\title{
Star-shaped quiver theories with flux
}

\author{
Shlomo S. Razamat ${ }^{1}$ and Brian Willett $\odot^{2}$ \\ ${ }^{1}$ Department of Physics, Technion, Haifa 32000, Israel \\ ${ }^{2}$ Kavli Institute for Theoretical Physics University of California, Santa Barbara, California 93106, USA
}

(Received 21 November 2019; accepted 22 January 2020; published 13 March 2020)

\begin{abstract}
We study the compactification of the six-dimensional (6D) $\mathcal{N}=(2,0)$ superconformal field theory on the product of a Riemann surface with flux and a circle. On the one hand, this can be understood by first reducing on the Riemann surface, giving rise to $4 \mathrm{D} \mathcal{N}=1$ and $\mathcal{N}=2$ class $\mathcal{S}$ theories, which we then reduce on $S^{1}$ to get 3D $\mathcal{N}=2$ and $\mathcal{N}=4$ class $\mathcal{S}$ theories. On the other hand, we may first compactify on $S^{1}$ to get the $5 \mathrm{D} \mathcal{N}=2$ Yang-Mills theory. By studying its reduction on a Riemann surface, we obtain a mirror dual description of $3 \mathrm{D}$ class $\mathcal{S}$ theories, generalizing the star-shaped quiver theories of Benini, Tachikawa, and Xie. We comment on some global properties of the gauge group in these reductions and test the dualities by computing various supersymmetric partition functions.
\end{abstract}

DOI: 10.1103/PhysRevD.101.065004

\section{INTRODUCTION}

Studying quantum field theories (QFTs) on compact spaces often leads to insights into complicated dynamics of lower dimensional theories. For example, many dualities between lower dimensional superconformal field theories (SCFTs) can be deduced from dualities connecting higher dimensional theories. A particular example is understanding $\mathcal{N}=2$ dualities in three-dimensional (3D) starting from $4 \mathrm{D} \mathcal{N}=1$ dual theories compactified on a circle [1-5]. Another example is the many insights derived in recent years, following the seminal paper [6], about strong coupling dynamics of $4 \mathrm{D} \mathcal{N} \geq 1$ by understanding them as compactifications of 6D SCFTs on Riemann surfaces. This has led to improved understanding of dualities and the emergence of symmetry in many examples of 4D SCFTs (see, e.g., [7-12] and references therein). Importantly, the 6D SCFTs here do not have at the moment a useful description in terms of fields and Lagrangians; see [13] for a nice review.

When one considers compactifications of higher dimensional quantum field theory, the resulting lower dimensional model is typically not given just by the Kaluza-Klein reduction of the higher dimensional fields with the same types of interactions. One can understand the problem as follows. In such a setup, there are two limits involved: first, we have the computation of the path integral, and second, we have a geometric parameter, the size of the compact part

Published by the American Physical Society under the terms of the Creative Commons Attribution 4.0 International license. Further distribution of this work must maintain attribution to the author(s) and the published article's title, journal citation, and DOI. Funded by SCOAP. of the geometry, which we take to be small. These two limits need not commute. A concrete example of this is that of taking 4D theories on a circle: the fact that some of the 4D symmetries are anomalous leads to novel interaction terms in the effective 3D theory, which explicitly break the anomalous symmetry $[4,5]$. Compactifying $3 \mathrm{D}$ theories to 2D leads to many complications of this sort [14], and similarly for $4 \mathrm{D}$ models reduced to $2 \mathrm{D}[15,16]$, and it is fair to say that such reductions are not understood well enough.

In this paper, we will discuss some aspects of compactifications of 6D SCFTs down to 3D. We will not consider the compactifications on a generic 3D manifold, as was done for example in [17-19], but rather on a geometry which has the structure of (punctured) Riemann surface times a circle. There are two ways to view such a compactification. We can either first try to understand the reduction on a circle down to 5D and then a subsequent compactification to $3 \mathrm{D}$, or first compactify to $4 \mathrm{D}$ and then to $3 \mathrm{D}$. The former way has the advantage that, although the 6D theories are not given in terms of Lagrangians, often when compactified on a circle (possibly with holonomies for various symmetries), they possess an effective 5D description in terms of fields. This 5D description then can be directly used to understand the further compactification down to 3D. This will still be a nontrivial task, following the comments we made above; however, we will be able to partially fix the 3D field content and action in terms of the 5D Lagrangian and the flavor symmetry background on the Riemann surface.

A useful set of tools in the analysis of dimensional reduction in supersymmetric field theories have been the supersymmetric partition functions on product manifolds. In this case, we can understand the reduction from $5 \mathrm{D}$ to $3 \mathrm{D}$ 
using the $S_{b}^{3} \times \Sigma_{g}$ partition function, studied in [20], which can be interpreted as the $S_{b}^{3}$ partition function of the dimensionally reduced theory. This partition function is closely related to the twisted index studied in lower dimensions [21-24], which has been used to study similar reductions from $4 \mathrm{D}$ to $2 \mathrm{D}$ in [15].

On the other hand, the approach where we first compactify to $4 \mathrm{D}$ often leads to theories which are rather complicated and typically do not have known Lagrangian descriptions. This makes it harder to understand the further compactifications to 3D. In some well-behaved cases, we can nevertheless study this reduction and the resulting 3D models. We will then discuss how the two different orders of compactification are related to each other. In particular, this leads to two different dual descriptions of the 3D SCFT obtained in the reduction.

Although we expect this procedure to apply to more general 6D models, we will concretely discuss compactifications of $A_{N-1}(2,0) \mathrm{SCFT}$ and give explicit details for the $A_{1}$ case, as here the 4D intermediate step is particularly simple. For the cases preserving $\mathcal{N}=4$ supersymmetry in $3 \mathrm{D}$, the dual descriptions are the mirror dualities of [25], which take the form of a "star-shaped quiver" with a central $S U(N) / \mathbb{Z}_{N}$ node. More generally, we may compactify with a flux, $\mathfrak{n}$, for the $S p(1)_{F}$ 6D flavor symmetry on the Riemann surface, which leads to an $\mathcal{N}=2$ model in $3 \mathrm{D}$, and here we find simple dual descriptions with a number of adjoint chiral multiplets for the central node which is linear in the flux, $\mathfrak{n}$.

The paper is organized as follows. In Sec. II, we discuss reductions on a Riemann surfaces with flux of general $\mathcal{N}=15 \mathrm{D}$ SCFTs and in particular the maximally supersymmetric (SYM), which is a circle reduction of the $6 \mathrm{D}$ $(2,0)$ theory. In Sec. III, we then analyze the reductions of 4D $\mathcal{N}=1$ class $\mathcal{S}$ theories [6,26], which are obtained by compactifying the $(2,0)$ theory on a Riemann surface with flux on a circle. Next, the two orders of the reduction are compared in Sec. IV and the ensuing dualities are discussed. In Sec. V, we discuss technical checks of the dualities using various supersymmetric partition functions. We comment on our results and possible generalizations in Sec. VI. Appendix collects several useful properties of the $T[S U(N)]$ models.

\section{REDUCTION OF 5D $\mathcal{N}=1$ GAUGE THEORIES ON $\Sigma_{g}$}

In this section, we describe some general aspects of the reduction of a $5 \mathrm{D} \mathcal{N}=1$ gauge theory on a Riemann surface, $\Sigma_{g}$, which in general gives rise to a $3 \mathrm{D} \mathcal{N}=2$ theory. We start by decomposing the fields into modes on $\Sigma_{g}$ and analyzing the resulting spectrum of fields in 3D. We then analyze the same problem from the perspective of the $S_{b}^{3} \times \Sigma_{g}$ partition function, computed in [20], and point out some new features that arise in this analysis. After this general analysis, we focus on the case of the $5 \mathrm{D} \mathcal{N}=2$ SYM theory, which will be our main example.

\section{A. Modes on $\boldsymbol{\Sigma}_{g}$}

A general $5 \mathrm{D} \mathcal{N}=1$ gauge theory has an $\operatorname{Sp}(1)_{R}$ R-symmetry and gauge and flavor symmetries, $G$ and $G_{F}$. The matter content consists of a vector multiplet, $V$, in the adjoint representation of $G$, and hypermultiplets, $H_{i}$, which come in a pseudoreal representation, $R_{i} \otimes S_{i}$, of $G \times G_{F}$. The field content and representations of these multiplets are as follows:

\begin{tabular}{lccccc}
\hline Multiplet & Field & $S O(5)_{E}$ & $S p(1)_{R}$ & $G$ & $G_{F}$ \\
\hline$V$ & $\sigma$ & 1 & 1 & $\operatorname{Adj}$ & 1 \\
& $\Lambda$ & 4 & 2 & $\operatorname{Adj}$ & 1 \\
& $A_{\mu}$ & 5 & 1 & $\operatorname{Adj}$ & 1 \\
$H_{i}$ & $Q_{i}$ & 1 & 2 & $R_{i}$ & $S_{i}$ \\
& $\Psi_{i}$ & 4 & 1 & $R_{i}$ & $S_{i}$ \\
\hline
\end{tabular}

Here we impose reality conditions on $\Lambda, Q_{i}$, and $\Psi_{i}$, which each live in a tensor product of two pseudoreal representations. The group $S O(5)_{E}$ is the group of rotations of the $5 \mathrm{D}$ Euclidean space.

We consider the theory on the spacetime $\mathbb{R}^{3} \times \Sigma_{g}$, and to preserve supersymmetry we perform a partial topological twist along $\Sigma_{g}$. This consists of introducing a background R-symmetry gauge field in the $U(1)_{R}$ maximal torus of $S p(1)_{R}$, which we identify with the spin connection on $\Sigma_{g}$. In addition, we may introduce arbitrary GNO fluxes for $G \times G_{F}$, which take values in the coweight lattices of these groups, and we denote these fluxes by $(\mathfrak{m}, \mathfrak{n})$. The symmetry which is unbroken in $3 \mathrm{D}$ after this twist consists of $U(1)_{R}$ and the commutant of $G$ and $G_{F}$ with the fluxes that we turn on. In principle, we should consider the contribution from all the dynamical gauge fluxes, $\mathfrak{m}$, but we will argue that in favorable cases only the sector with $\mathfrak{m}=0$ contributes, so for now we will specialize to this case.

Let us start by decomposing these fields into modes on $\Sigma_{g}$, each of which gives rise to a $3 \mathrm{D}$ field. First, we decompose into representations of $S O(3)_{E} \times U(1)_{\Sigma_{g}} \subset$ $S O(5)_{E}$, under which $4 \rightarrow 2_{ \pm 1}$ and $5 \rightarrow 3_{0} \oplus 1_{ \pm 2}$. Recall that (left/right-handed) fermions on $\Sigma_{g}$ are sections of the line bundle $\mathcal{O}( \pm(g-1))$, while one forms are sections of $\mathcal{O}( \pm 2(g-1))$. Thus, before the twist, the fields take values in the following sections of $\Sigma_{g}$ :

\begin{tabular}{lcccccc}
\hline Multiplet & Field & Bundle & $S O(3)_{E}$ & $U(1)_{R}$ & $G$ & $G_{F}$ \\
\hline$V$ & $\sigma$ & $\mathcal{O}(0)$ & 1 & 0 & Adj & 1 \\
& $\Lambda$ & $\mathcal{O}( \pm(g-1))$ & 2 & -1 & Adj & 1 \\
& $A_{\mu}$ & $\mathcal{O}(0)$ & 3 & 0 & Adj & 1 \\
& $A_{z}$ & $\mathcal{O}(2(g-1))$ & 1 & 0 & Adj & 1 \\
$H_{i}$ & $Q_{i}$ & $\mathcal{O}(0)$ & 1 & 1 & $R_{i}$ & $S_{i}$ \\
& $\Psi_{i}$ & $\mathcal{O}(g-1)$ & 2 & 0 & $R_{i}$ & $S_{i}$ \\
\hline
\end{tabular}


Now, we consider the effect of the twist. First, we look at the vector multiplet. Here only the $U(1)_{R}$ flux has an effect, and after the twist the fields behave as follows:

\begin{tabular}{ccccccc}
\hline Multiplet & Field & Bundle & $S O(3)_{E}$ & $U(1)_{R}$ & $G$ & $G_{F}$ \\
\hline$V$ & $\sigma$ & $\mathcal{O}(0)$ & 1 & 0 & Adj & 1 \\
& $\Lambda$ & $\mathcal{O}(0)$ & 2 & -1 & Adj & 1 \\
& $A_{\mu}$ & $\mathcal{O}(0)$ & 3 & 0 & Adj & 1 \\
& $A_{z}$ & $\mathcal{O}(2(g-1))$ & 1 & 0 & Adj & 1 \\
& $\Lambda_{z}$ & $\mathcal{O}(2(g-1))$ & 2 & 1 & Adj & 1
\end{tabular}

We see that $\left(\sigma, \Lambda, A_{\mu}\right)$ contain the fields of a $3 \mathrm{D} \mathcal{N}=2$ vector multiplet. In principle, we obtain such a multiplet for each mode on $\Sigma_{g}$; however, all of the nonholomorphic modes will pair up into long multiplets and decouple from BPS observables, and so we obtain a single $3 \mathrm{D} \mathcal{N}=2$ vector multiplet from the single (constant) zero mode of $\mathcal{O}(0)$. In addition, $\left(A_{z}, \Lambda_{z}\right)$ transform like a one form on $\Sigma_{g}$, and we expect $g$ holomorphic zero modes for this bundle, which leads to $g 3 \mathrm{D} \mathcal{N}=2$ adjoint chiral multiplets.

Next, consider the hypermultiplet, $H_{i}$. Recall this sits in a pseudoreal representation, $R_{i} \otimes S_{i}$, of $G \times G_{F}$, and so the weights come in pairs, $(\rho, \omega)$ and $(-\rho,-\omega)$, and let us consider the contribution from one such pair. Then these fields get an additional contribution to their flux of $\pm \omega(\mathfrak{n})$. The resulting field content after the twist is then as follows:

\begin{tabular}{lcccccc}
\hline Multiplet & Field & Bundle & $S O(3)_{E}$ & $U(1)_{R}$ & $G$ & $G_{F}$ \\
\hline$H_{i}$ & $Q_{i}$ & $\mathcal{O}(g-1+\omega(\mathfrak{n}))$ & 1 & 1 & $\rho$ & $\omega$ \\
& $\Psi_{i}$ & $\mathcal{O}(g-1+\omega(\mathfrak{n}))$ & 2 & 0 & $\rho$ & $\omega$ \\
& $\tilde{Q}_{i}$ & $\mathcal{O}(g-1-\omega(\mathfrak{n}))$ & 1 & 1 & $-\rho$ & $-\omega$ \\
& $\tilde{\Psi}_{i}$ & $\mathcal{O}(g-1-\omega(\mathfrak{n}))$ & 2 & 0 & $-\rho$ & $-\omega$
\end{tabular}

Here, the number of holomorphic sections of these bundles will depend on the precise metric and gauge connection we choose; however, this number is constrained by the Riemann-Roch index theorem, which states

$$
\begin{aligned}
& \operatorname{dim} H_{0}\left(\Sigma_{g}, \mathcal{O}(n)\right)-\operatorname{dim} H_{0}\left(\Sigma_{g}, \mathcal{O}(2(g-1)-n)\right) \\
& \quad=n+1-g .
\end{aligned}
$$

Thus, we find some number $N_{\rho, \omega}$ of $3 \mathrm{D} \mathcal{N}=2$ chiral multiplets transforming with weight $(\rho, \omega)$, and $N_{-\rho,-\omega}$ with weight $(-\rho,-\omega)$, subject to

$$
N_{\rho, \omega}-N_{-\rho,-\omega}=\omega(\mathfrak{n}) .
$$

Importantly, BPS observables, such as supersymmetric partition functions, will depend only on this difference; however, the precise field content cannot be determined by this analysis.
To summarize the analysis above, we found that

$$
5 \mathrm{D} \mathcal{N}=1 \text { vector multiplet }
$$

$\downarrow$ compactification on $\Sigma_{g}$ with flux $\mathfrak{n}$

$3 \mathrm{D} \mathcal{N}=2$ vector multiplet +

$g \times 3 \mathrm{D} \mathcal{N}=2$ adjoint chiral multiplets of R-charge zero

5D $\mathcal{N}=1$ hyper multiplet with weight $(\rho, \omega)$

$\downarrow$ compactification on $\Sigma_{g}$ with flux $\mathfrak{n}$

$N_{\rho, \omega} \times 3 \mathrm{D} \mathcal{N}=2$ chirals with weight $(\rho, \omega)$

$+N_{-\rho,-\omega} \times 3 \mathrm{D} \mathcal{N}=2$ chirals with weight $(-\rho,-\omega)$

both with R-charge 1 , and with $N_{\rho, \omega}-N_{-\rho,-\omega}=\omega(\mathfrak{n})$.

We emphasize that the above analysis was performed in the zero gauge flux sector. We comment below on the possibility of other gauge flux sectors contributing in 3D.

\section{B. The $S_{b}^{3} \times \Sigma_{g}$ partition function}

We can gain another perspective on the reduction by studying the $S_{b}^{3} \times \Sigma_{g}$ partition function, computed in [20]. Let us fix a $5 \mathrm{D} \mathcal{N}=1$ theory with semisimple gauge group $G$, and with matter in a representation $R$ of the gauge and flavor group, $G \times G_{F}{ }^{1}$ Then, from [20], the partition function on $S_{b}^{3} \times \Sigma_{g}$ may be written as $^{2}$

$$
\begin{aligned}
& Z_{S_{b}^{3} \times \Sigma_{g}}(\nu)_{\mathfrak{n}} \\
& =\frac{1}{\left|W_{G}\right|} \sum_{\mathfrak{m} \in \Lambda_{G}} \\
& \quad \times \int d u e^{2 \pi i \gamma T r(u \mathfrak{m})} \prod_{\alpha \in A d(G)^{\prime}} s_{b}(-i Q+\alpha(u))^{-\alpha(\mathfrak{m})+1-g} \\
& \quad \times \prod_{(\rho, \omega) \in R} s_{b}(\rho(u)+\omega(\nu))^{\rho(\mathfrak{m})+\omega(\mathfrak{n})} H^{g} Z_{\text {inst }}(u, \nu)_{\mathfrak{m}, \mathfrak{n}} .
\end{aligned}
$$

Here $s_{b}(x)$ is the double sine function (see, e.g., [27]), $u$ and $\mathfrak{m}$ parametrize the gauge vector multiplet, determining the real scalar and flux through $\Sigma_{g}$, respectively, and these parametrize the BPS locus we integrate over after applying localization. Similarly, $\nu$ and $\mathfrak{n}$ describe background vector

\footnotetext{
${ }^{1}$ One could also include a 5D Chern-Simons term, but we will not consider this case here.

${ }^{2}$ We refer to [20] for more details and conventions.
} 
multiplets coupled to the flavor symmetry and are parameters of the partition function. In addition, we have defined $\gamma=-\frac{2 \pi Q}{g_{5}{ }^{2}}$, where $Q=\frac{b+b^{-1}}{2}$ and $g_{5}$ are the 5D gauge coupling, and $\operatorname{Ad}(G)^{\prime}$ refers to the nonzero roots of $G$. The perturbative Hessian, $H$, is given by

$$
\begin{aligned}
H= & \operatorname{det}_{a b}\left(\gamma K_{a b}+\sum_{(\rho, \omega) \in R} \rho_{a} \rho_{b} \frac{s_{b}^{\prime}(\rho(u)+\omega(\nu))}{s_{b}(\rho(u)+\omega(\nu))}\right. \\
& \left.-\sum_{\alpha \in \operatorname{Ad}(G)^{\prime}} \frac{s_{b}^{\prime}(-i Q+\alpha(u))}{s_{b}(-i Q+\alpha(u))}\right) .
\end{aligned}
$$

Finally, $Z_{\text {inst }}(u, \nu)_{\mathfrak{m}, \mathfrak{n}}$ refers to the instanton contribution to the partition function, which we do not write explicitly here.

This partition function has a similar structure to the $S^{3}$ partition function [27-29] of a 3D $\mathcal{N}=2$ gauge theory, including the expected one-loop contributions of the chiral and vector multiplets, expressed through the double sine function, $s_{b}(x)$. However, there are two important differences. First, there is an infinite sum over flux sectors, $\mathfrak{m}$, on $\Sigma_{g}$. We may tentatively interpret this as implying the system is described by an infinite direct sum of 3D $\mathcal{N}=2$ theories. Similar direct sums have appeared in other examples of dimensional reduction of gauge theories, e.g., in [14,30,31]. In addition, there are extra factors related to the fermion zero modes and instanton contributions, which are not straightforwardly interpreted in terms of the $S_{b}^{3}$ partition function of ordinary $3 \mathrm{D} \mathcal{N}=2$ gauge theories, implying this would have to be a more exotic $3 \mathrm{D} \mathcal{N}=2$ theory.

Below, we will focus on a special case where the 3D interpretation is more straightforward. There is a class of $5 \mathrm{D}$ gauge theories which are believed to have a UV completion as a $6 \mathrm{D} \mathcal{N}=(1,0)$ SCFT. Specifically, there is an emergent circle, whose radius is proportional to the 5D gauge coupling, $\beta \sim g_{5}$. In these cases, the partition function above can be reinterpreted as the $S_{b}^{3} \times \Sigma_{g} \times S_{\beta}^{1}$ partition function of this 6D theory, or equivalently, as the $S_{b}^{3} \times S_{\beta}^{1}$ partition function of the $4 \mathrm{D}$ theory obtained by compactification on $\Sigma_{g}$. If we now consider the limit $\beta \sim g_{5} \rightarrow 0$, we may interpret this as the dimensional reduction of the $4 \mathrm{D}$ theory, which we expect to give an ordinary 3D theory.

Several things happen in this limit of the partition function above. First, and most importantly, we expect that the instanton contribution drastically simplifies and can be essentially ignored. In fact, we will argue below that it contributes an overall factor which can be related to the Cardy behavior of the 4D index as $\beta \rightarrow 0$.

Next, note that for $\mathfrak{m} \neq 0$, because of the $e^{2 \pi i \gamma T r(u \mathfrak{m})}$ factor, and since $\gamma \sim \beta^{-1}$ is taken very large, the integrand is rapidly oscillating in at least one direction in the complex $u$ plane, and so by the Riemann-Lebesgue lemma, we expect its contribution to vanish as $\gamma^{-1}$ is taken small. In addition, we note that the first term in (4) is dominant, and so we may approximate

$$
H \approx \gamma^{r_{G}},
$$

where $r_{G}$ is the rank of $G$. More precisely, these two statements only follow provided the integrand is bounded at infinity. But, as argued in Sec. 4.2.2 of [20], this is true precisely for those $5 \mathrm{D}$ theories which have $6 \mathrm{D}$ uplifts.

With these assumptions, we may approximate

$$
\begin{aligned}
Z_{S_{b}^{3} \times \Sigma_{g}}(\nu)_{\mathfrak{n}} \underset{g_{5} \rightarrow 0}{\approx} & \frac{\gamma^{g r_{G}}}{\left|W_{G}\right|} \int d u \prod_{\alpha \in A d(G)^{\prime}} s_{b}(-i Q+\alpha(u))^{1-g} \\
& \times \prod_{(\rho, \omega) \in R} s_{b}(\rho(u)+\omega(\nu))^{\omega(\mathfrak{n})}
\end{aligned}
$$

In this form, the partition function looks very similar to the $S_{b}^{3}$ partition function of a certain $3 \mathrm{D} \mathcal{N}=2$ gauge theory. In fact, we claim this theory is precisely the one we were led to by the analysis of the previous subsection.

To see this, note that the first product in the integrand can be interpreted as the contribution of a $3 \mathrm{D} \mathcal{N}=2$ vector multiplet, along with $g$ adjoint chiral multiplets of R-charge zero, as in (3). The latter contribute oppositely to the vector, so their total contribution appears as a single factor raised to the power $1-g$. To be precise, the adjoint chiral multiplets have an additional contribution from the $g r_{G}$ Cartan elements, which do not depend on the gauge variable, $u$. Since they also have R-charge zero, strictly speaking their contribution diverges. However, we may schematically identify this divergence with the $\gamma^{g r_{G}}$ prefactor, which is also diverging in this limit, and we note the exponent matches the number of Cartan elements.

Next, we look at the second product in the integrand in (6). Using the basic identity,

$$
s_{b}(u)=s_{b}(-u)^{-1},
$$

we see that we may interpret this as the contribution of $N_{\rho, \omega}$ chiral multiplets of weights $(\rho, \omega)$ along with $N_{-\rho,-\omega}$ of weight $(-\rho,-\omega)$, provided that

$$
N_{\rho, \omega}-N_{-\rho,-\omega}=\omega(\mathfrak{n}),
$$

precisely as in (4).

Thus, under the assumptions above which led us to argue the partition function truncates to the zero flux sector, we see the analysis of the previous subsection and that of the $S_{b}^{3} \times \Sigma_{g}$ partition function lead to the same result.

\section{Cardy scaling}

From the results of [32], we expect the 3D reduction of the $4 \mathrm{D}$ index to behave in the $\beta \rightarrow 0$ limit as 


$$
\lim _{\beta \rightarrow 0} \mathcal{I}\left(p, q, \mu_{i}\right)=\exp \left(-\frac{\pi}{6 \beta}\left(Q \mathcal{A}^{R}+\mathcal{A}^{\alpha} \nu_{\alpha}\right)\right) Z_{S_{b}^{3}}\left(\nu_{i}\right),
$$

where $\mathcal{A}^{R}$ and $\mathcal{A}^{\alpha}$ are the linear anomalies of the R-symmetry and flavor symmetries, respectively. Thus, while above we have found the expected finite piece, we did not observe the divergent "Cardy scaling." We expect this contribution will arise from the instanton contribution we have so far ignored. We will see this more explicitly when we consider the $5 \mathrm{D} \mathcal{N}=2$ theory next.

\section{Reduction of the 5D $\mathcal{N}=2$ SYM theory}

Our main example in this paper will be the $5 \mathrm{D} \mathcal{N}=2$ Yang-Mills theory with gauge group $G$, which we will usually take to be $S U(N)$. This has an $S p(2)_{R}$ symmetry, but in the $\mathcal{N}=1$ language used above, this decomposes to $S p(1)_{R} \times S p(1)_{F}$, with a single $5 \mathrm{D} \mathcal{N}=1$ vector multiplet and a $5 \mathrm{D} \mathcal{N}=1$ hypermultiplet in the representation (Adj, 2) of $G \times S p(1)_{F}$.

Our main interest in this example is due to the fact that it admits a UV completion as the $6 \mathrm{D} \mathcal{N}=(2,0)$ SCFT compactified on a circle. Then the $3 \mathrm{D}$ reduction of this $5 \mathrm{D}$ theory on $\Sigma_{g}$ may be alternatively described in terms of first compactifying the 6D SCFT on a Riemann surface with flux $\mathfrak{n}$ for the $S p(1)_{F}$ flavor symmetry, obtaining in general a $4 \mathrm{D} \mathcal{N}=1$ class $\mathcal{S}$ theory $[7,8,33]$ (see also [34-36]), ${ }^{3}$ and then dimensionally reducing this on a circle. Comparing the theories obtained by these two methods can then lead to nontrivial three-dimensional dualities. We will return to the $4 \mathrm{D}$ class $\mathcal{S}$ theories and their reduction in the next section.

\section{Reduction on $\Sigma_{g}$}

When compactifying the $5 \mathrm{D} \mathcal{N}=2$ theory on $\Sigma_{g}$, we may also include a flux, $\mathfrak{n} \in \mathbb{Z}$, for the $S p(1)_{F}$ flavor symmetry, and so we expect a family of theories labeled by $\mathfrak{n}$ and $g$. Let us first analyze this reduction in terms of the modes on $\Sigma_{g}$, as in Sec. II A. We find the field content in 3D shown in Table I. Here the numbers, $\ell$ and $\tilde{\ell}$, of adjoint chiral multiplets, $\Psi$ and $\tilde{\Psi}$, cannot be determined individually, but satisfy

$$
\ell-\tilde{\ell}=\mathfrak{n} .
$$

Let us first consider an important special case, which is $\mathfrak{n}=g-1$. This can be interpreted as performing the topological twist on $\Sigma_{g}$ using the R-symmetry,

$$
R_{+}=R+F
$$

\footnotetext{
${ }^{3}$ In the notation of [33], $\mathfrak{n}=\frac{p-q}{2}$, as discussed below.
}

TABLE I. Matter content of 3D reduction of 5D $\mathcal{N}=2 \mathrm{SYM}$. Here $V$ denotes a 3D $\mathcal{N}=2$ vector multiplet and the remaining multiplets are chiral multiplets, where the $U(1)_{R}$ charge refers to that of the scalar component.

\begin{tabular}{lcccc}
\hline \hline Multiplet & Number & $U(1)_{R}$ & $G$ & $U(1)_{F}$ \\
\hline$V$ & 1 & $\ldots$ & Adj & 0 \\
$\Omega_{a}$ & $g$ & 0 & Adj & 0 \\
$\Psi_{b}$ & $\ell$ & 1 & Adj & 1 \\
$\tilde{\Psi}_{c}$ & $\tilde{\ell}$ & 1 & Adj & -1 \\
\hline \hline
\end{tabular}

This is the same twist used to define $4 \mathrm{D} \mathcal{N}=2$ class $\mathcal{S}$ theories, and so we expect the 3D theories obtained here to be equivalent to their dimensional reduction. In this case, it is natural to take the background gauge field equal to the spin connection on $\Sigma_{g}$, and in this case the fields $\Psi$ and $\tilde{\Psi}$ are sections of $\mathcal{O}(2(g-1))$ and $\mathcal{O}(0)$, respectively, which can be identified with one forms and scalars. Then we are justified in taking

$$
\ell=g, \quad \tilde{\ell}=1 .
$$

Then we see the fields can be organized into the field content of 3D $\mathcal{N}=4$ theory, namely,

$$
\begin{aligned}
\left(V, \tilde{\Psi}_{1}\right) & \rightarrow 3 \mathrm{D} \mathcal{N}=4 \text { vector multiplet, } \\
\left(\Omega_{a}, \Psi_{a}\right) & \rightarrow g \times 3 \mathrm{D} \mathcal{N}=4 \text { adjoint hypermultiplets. }
\end{aligned}
$$

Let us check if the symmetries act as expected for a 3D $\mathcal{N}=4$ theory, whose symmetry group is $S L(2, \mathbb{R})_{\text {rot }} \times$ $S U(2)_{H} \times S U(2)_{C}$. Decomposing the R-symmetry under the $U(1)_{H} \times U(1)_{C}$ maximal torus, and allowing also a $U(1)_{f}$ flavor symmetry to act on the hypermultiplet, we see the expected behavior is as follows:

\begin{tabular}{ccccccc}
\hline $\mathcal{N}=4$ & $\mathcal{N}=2$ & Field & $S L(2, \mathbb{R})_{\text {rot }}$ & $U(1)_{H}$ & $U(1)_{C}$ & $U(1)_{f}$ \\
\hline Vector & $V$ & $\sigma$ & 1 & 0 & 0 & 0 \\
& & $\Lambda$ & 2 & -1 & -1 & 0 \\
& & $A_{\mu}$ & 3 & 0 & 0 & 0 \\
& $\Phi$ & $\phi$ & 1 & 0 & 2 & 0 \\
Hyper & $Q$ & $\psi_{\phi}$ & 2 & -1 & 1 & 0 \\
& $q$ & 1 & 1 & 0 & 1 \\
& $\tilde{Q}$ & $\psi$ & 2 & 0 & -1 & 1 \\
& & $\tilde{\psi}$ & 1 & 1 & 0 & -1 \\
& & & 2 & 0 & -1 & -1
\end{tabular}

On the other hand, we can consider the $U(1)_{R} \times U(1)_{F}$ symmetry acting on the fields obtained from $5 \mathrm{D}$. Here we include also the charges under a $U(1)_{f}$ flavor symmetry, which sits inside the $U(g)$ symmetry acting on the fields $\left(\Omega_{a}, \Psi_{a}\right)$, which is a hidden symmetry from the 5D point of view. 


\begin{tabular}{lccccc}
\hline Multiplet & Field & $S O(3)_{E}$ & $U(1)_{R}$ & $U(1)_{F}$ & $U(1)_{f}$ \\
\hline$V$ & $\sigma$ & 1 & 0 & 0 & 0 \\
& $\Lambda$ & 2 & -1 & 0 & 0 \\
& $A_{\mu}$ & 3 & 0 & 0 & 0 \\
$\Omega_{a}$ & $A_{z}$ & 1 & 0 & 0 & 1 \\
\multirow{3}{*}{$\Psi_{b}$} & $\Lambda_{z}$ & 2 & -1 & 0 & 1 \\
\multirow{2}{*}{$\tilde{\Psi}_{1}$} & $Q_{z}$ & 1 & 1 & 1 & -1 \\
& $\Psi_{z}$ & 2 & 0 & 1 & -1 \\
& $\tilde{Q}$ & 1 & 1 & -1 & 0 \\
& $\tilde{\Psi}$ & 2 & 0 & -1 & 0 \\
\hline
\end{tabular}

Then, we see the charges agree provided we identify

$$
H=\left\{\begin{array}{ll}
R+F & g=0 \\
R+F+f & g>0
\end{array}, \quad C=R-F .\right.
$$

Interestingly, to identify these symmetries, we see that for $g>0$, we must admix a flavor symmetry which is hidden from the $5 \mathrm{D}$ point of view. Note that this is very reminiscent of Gaiotto-Witten "bad" theories having hidden IR symmetry [37].

As mentioned above, we may also interpret this theory as the $S^{1}$ reduction of a $4 \mathrm{D} \mathcal{N}=2$ class $\mathcal{S}$ theory, obtained by compactifying the $6 \mathrm{D} \mathcal{N}=(2,0)$ theory on a Riemann surface. The 3D reduction of the class $\mathcal{S}$ theory associated to a Riemann surface has a dual description, found in [25], as a so-called star-shaped quiver. In the present case, with no flavor punctures, this is a 3D $\mathcal{N}=4$ theory with $g$ adjoint hypermultiplets. This is precisely the description we have found above, providing an alternative derivation of their result.

Note that when we twist by the $U(1)_{R_{+}} \subset S p(1)_{R} \times$ $S p(1)_{F} \subset S p(2)_{R}$ symmetry in $5 \mathrm{D}$, the commutant is an $S U$ (2) subgroup, with Cartan $R_{-}=R-F$, which, using (13), we can identify with the $S U(2)_{C}$ symmetry. On the other hand, if we consider this 5D theory as a $6 \mathrm{D}$ theory compactified on a circle, this $S U(2)$ commutant becomes the $S U(2)_{R}$ symmetry of the resulting $4 \mathrm{D} \mathcal{N}=2$ class $\mathcal{S}$ theory. In the usual convention, this $S U(2)_{R}$ symmetry becomes, upon dimensional reduction, the $S U(2)_{H}$ symmetry of the resulting 3D $\mathcal{N}=4$ class $\mathcal{S}$ theory. The fact that it acts as an $S U(2)_{C}$ symmetry above reflects the fact that this description should be considered a "mirror dual" of the $3 \mathrm{D} \mathcal{N}=4$ class $\mathcal{S}$ theory.

For future reference, we will find it useful to define $\mathcal{N}=2^{*} U(1)_{r}$ symmetry and $U(1)_{t}$ flavor symmetries as follows:

$$
\begin{gathered}
r=\frac{1}{2}(H+C)=\left\{\begin{array}{ll}
R & g=0 \\
R+\frac{f}{2} & g>0
\end{array},\right. \\
T=\frac{1}{2}(C-H)=\left\{\begin{array}{ll}
-F & g=0 \\
-F-\frac{f}{2} & g>0
\end{array} .\right.
\end{gathered}
$$

Here we have defined the $U(1)_{t}$ flavor symmetry with a sign relative to the usual convention. This is in anticipation of comparison to the dimensional reduction of 4D models, where, given the previous paragraph, we expect the usual $U(1)_{t}$ symmetry to map to the one with a flip of sign defined above.

The above argument can be generalized for arbitrary flux, $\mathfrak{n}$, for the $U(1)_{F}$ symmetry, which will in general lead to a $3 \mathrm{D} \mathcal{N}=2$ theory. These theories may alternatively be obtained by dimensional reduction of $4 \mathrm{D} \mathcal{N}=1$ class $\mathcal{S}$ theories associated to compactification on a Riemann surface with flux $[7,8,33]$, which we describe in more detail in the next section. For general $\mathfrak{n}$, the matter content can be summarized by Table I above, and we note the charges are compatible with the superpotential

$$
W=\sum \Omega_{a} \Psi_{b} \tilde{\Psi}_{c}
$$

where the sum is over any subset of the allowed values of the indices. For example, in the $\mathcal{N}=4$ case, where $\mathfrak{n}=g-1$, the superpotential in (15) may be taken as

$$
W=\sum_{a=1}^{g} \Omega_{a} \tilde{\Psi}_{1} \Psi_{a}
$$

which is the appropriate $3 \mathrm{D} \mathcal{N}=4$ superpotential. A similar statement holds for the case $\mathfrak{n}=-(g-1)$, with the roles of $\Psi$ and $\tilde{\Psi}$ exchanged.

Another interesting example is the case $\mathfrak{n}=0$. Then $\ell=\tilde{\ell}$, so the adjoint chiral multiplets come in $\ell$ pairs, and, although we cannot fix them individually, we can see that each such pair forms a doublet of the $S U(2)_{F}$ symmetry, which remains unbroken in this case. The $4 \mathrm{D}$ parents of these theories are the so-called "Sicilian" 4D $\mathcal{N}=1$ theories [7].

To summarize, we find that the 3D theory corresponding to the compactification of the $6 \mathrm{D} \mathcal{N}=(2,0) A_{N-1}$ theory on a Riemann surface of genus $g$ and with flux $\mathfrak{n}$ for the $U(1)_{F} \subset S U(2)_{F}$ flavor symmetry is described by

$$
\begin{aligned}
& \mathfrak{s} \mathfrak{t}(N) \text { gauge theory with } g, \ell \text {, and } \tilde{\ell} \\
& \text { adjoint chirals of } U(1)_{F} \text { charge } 0,1 \text { and }-1 \\
& \text { where } \ell-\tilde{\ell}=\mathfrak{n} .
\end{aligned}
$$

In Sec. II D below. we will see that the global form of the gauge group is naturally taken to be $S U(N) / \mathbb{Z}_{N}$,

$$
S_{b}^{3} \times \Sigma_{g} \text { partition function. }
$$

Let us now briefly reconsider the above analysis using the $S_{b}^{3} \times \Sigma_{g}$ partition function. In this case, the perturbative partition function is given by 


$$
\begin{aligned}
Z_{S_{b}^{3} \times \Sigma_{g}}^{\mathcal{N}=2, \text { pert }}(\nu)_{\mathfrak{n}} \\
=\frac{1}{\left|W_{G}\right|} \sum_{\mathfrak{m} \in \Lambda_{G}} \int d u e^{2 \pi i \gamma \operatorname{Tr}(u \mathfrak{m})} \prod_{\alpha \in \operatorname{Ad}(G)} s_{b}(\alpha(u)+\nu)^{\alpha(\mathfrak{m})+\mathfrak{n}} \\
\quad \times \prod_{\alpha \in A d(G)^{\prime}} s_{b}(-i Q+\alpha(u))^{-\alpha(\mathfrak{m})+1-g} H^{g} .
\end{aligned}
$$

Here $\nu$ and $\mathfrak{n}$ are the mass and flux, respectively, for the $S U(2)_{F}$. Also, $\gamma=-\frac{2 \pi Q}{g_{5}^{2}}$, where $Q=\frac{b+b^{-1}}{2}$.

Now, we consider the limit $g_{5} \rightarrow 0$, or equivalently, $\gamma \rightarrow \infty$. As above, in this limit, we expect to be justified in considering only the perturbative contribution and the $\mathfrak{m}=0$ term in the sum over fluxes, and we find

$$
\begin{aligned}
Z_{S_{b}^{3} \times \Sigma_{g}}^{\mathcal{N}=2, \text { pert }}(\nu)_{\mathfrak{n}} & \underset{g_{5} \rightarrow 0}{\approx} \frac{\gamma^{g r_{G}}}{\left|W_{G}\right|} \int d u \prod_{\alpha \in \operatorname{Ad}(G)} s_{b}(\alpha(u)+\nu)^{\mathfrak{n}} \\
& \times \prod_{\alpha \in \operatorname{Ad}(G)^{\prime}} s_{b}(-i Q+\alpha(u))^{1-g}
\end{aligned}
$$

Let us first consider the case $\mathfrak{n}=g-1(\mathfrak{n}=-(g-1)$ is analogous), corresponding to reduction preserving 3D $\mathcal{N}=4$ supersymmetry. Then, we may write

$$
\begin{aligned}
Z_{S_{b}^{3} \times \Sigma_{g}}^{\mathcal{N}=2 \text { pert }}(\nu)_{\mathfrak{n}=g-1} \underset{\gamma \rightarrow \infty}{\approx} & \frac{\gamma^{g r_{G}} s_{b}(\nu)^{r_{G}(g-1)}}{\left|W_{G}\right|} \\
& \times \int d u \prod_{\alpha \in \operatorname{Ad}(G)^{\prime}}\left(\frac{s_{b}(-i Q+\alpha(u))}{s_{b}(\alpha(u)+\nu)}\right)^{1-g} .
\end{aligned}
$$

Let us compare this to the $S_{b}^{3}$ partition function of the starshaped quiver of [7]. Here, we use the standard $U(1)_{r}$ and $U(1)_{t}$ symmetries, as in (14), as well as the $U(g)$ flavor symmetry, and denote their fugacities by $\tau$ and $\mu_{i}$, $i=1, \ldots, g$, respectively, giving

$$
\begin{aligned}
& Z_{S_{b}^{3}}^{\mathrm{SSQ}}\left(\mu_{i}, \tau\right) \\
& =\frac{1}{\left|W_{G}\right|} \int d u \prod_{\alpha \in A d(G)^{\prime}} s_{b}(-i Q+\alpha(u)) \\
& \quad \times \prod_{\alpha \in \operatorname{Ad}(G)}\left(s_{b}(\tau+\alpha(u)) \prod_{i=1}^{g} s_{b}\left(\frac{i Q}{2}-\frac{\tau}{2} \pm \mu_{i}+\alpha(u)\right)\right),
\end{aligned}
$$

where the first two factors in the integrand come from the $3 \mathrm{D} \mathcal{N}=4$ vector multiplet, and the remaining factors come from the adjoint hypers. This $U(g)$ symmetry is accidental from the point of view of the 5D theory, and so the limit of the 4D index does not give us access to the full symmetry, but places us at a particular point in the space of real mass parameters. In fact, following the analysis that led to (13), one finds that we should identify

$$
\tau=-\nu, \quad \mu_{i}=\frac{-i Q+\nu}{2} .
$$

Then, we find

$$
\begin{aligned}
Z_{S_{b}^{3}}^{\mathrm{SSQ}}= & s_{b}(\nu)^{r_{G}(g-1)} s_{b}(i Q)^{g r_{G}} \\
& \times \frac{1}{\left|W_{G}\right|} \int d u \prod_{\alpha \in A d(G)^{\prime}}\left(\frac{s_{b}(-i Q+\alpha(u))}{s_{b}(\nu+\alpha(u))}\right)^{1-g} .
\end{aligned}
$$

This expression is somewhat formal for $g>0$, as $s_{b}(x)$ has a simple pole at $x=i Q$. However, comparing to (20), we see the finite pieces precisely agree, and the divergences also formally match if we identify $s_{b}(i Q) \sim \gamma \rightarrow \infty$. A similar analysis can be carried out for more general flux, $\mathfrak{n}$, and we find the $S_{b}^{3}$ partition function of the $3 \mathrm{D} \mathcal{N}=2$ theory described above.

\section{Cardy scaling and the Schur limit}

As mentioned above, we expect the instantons, which we have so far ignored, will contribute the expected Cardy scaling of the $4 \mathrm{D}$ index as $\beta \rightarrow 0$, as in (9). First, to see what result we expect, the anomaly polynomial of the general $4 \mathrm{D} \mathcal{N}=1$ theory above was computed in [33], and in particular,

$$
\operatorname{Tr} \mathcal{R}=(g-1) r_{G}(1+z \epsilon),
$$

where $z=\frac{\mathfrak{n}}{(g-1)}$ and $\epsilon$ is the mixing parameter of the R-symmetry with the $U(1)_{t}$ flavor symmetry. After adapting to our notation, this leads to a predicted Cardy scaling of

$$
\exp \left(-\frac{\pi \gamma}{12}(N-1)\left(\left(b+b^{-1}\right)(g-1)-2 i \nu \mathfrak{n}\right)\right) .
$$

The $S_{b}^{3} \times \Sigma_{g}$ partition function of the $\mathcal{N}=2$ SYM theory admits a special limit with enhanced supersymmetry, called the "Schur limit" in [20] due to its relation to the Schur limit of the 4D index [38]. This corresponds to setting

$$
\nu=\frac{i}{2}\left(b-b^{-1}\right)
$$

In this limit, the partition function greatly simplifies, and one can compute the instanton contribution is given by

$$
Z_{\text {inst }}(\nu)_{\mathfrak{n}}=\eta(z)^{(N-1)(g-1+\mathfrak{n})} \eta(\bar{z})^{(N-1)(g-1-\mathfrak{n})},
$$

where

$$
z=e^{-2 \pi b \gamma}, \quad \bar{z}=e^{-2 \pi b^{-1} \gamma} .
$$

Now, the limit $\gamma \rightarrow \infty$ corresponds to taking both $z$ and $\bar{z}$ to 0 , and in this limit, we have 


$$
\eta(z) \approx e^{-\frac{\pi b_{\gamma}}{12}}, \quad \eta(\bar{z}) \approx e^{-\frac{\pi b^{-1} \gamma}{12}}, \quad \gamma \rightarrow \infty
$$

Putting this together, we find a leading divergence of

$$
\exp \left(-\frac{\pi \gamma}{12}(N-1)\left(\left(b+b^{-1}\right)(g-1)+\left(b-b^{-1}\right) \mathfrak{n}\right)\right)
$$

which agrees with (25) in this limit. It would be interesting to extend this analysis to more general values of the flavor fugacity.

\section{Punctures}

Above we derived a dual description for the $4 \mathrm{D} \mathcal{N}=1$ theory associated to the compactification of the $6 \mathrm{D} \mathcal{N}=$ $(2,0)$ theory on a Riemann surface with flux, but with no punctures. Let us briefly comment on extending the above result to the case with punctures.

After reducing on the $S^{1}$ factor, this corresponds to a codimension 2 defect in the 5D $\mathcal{N}=2$ SYM theory. Then, as described in the context of $4 \mathrm{D} \mathcal{N}=2$ compactifications in $[25,39,40]$, this defect can be understood by coupling the $5 \mathrm{D}$ degrees of freedom to the $3 \mathrm{D} \mathcal{N}=4$ theory $T_{\rho}[G]$ of [37], which describes a boundary condition of the 4D $\mathcal{N}=$ 4 theory. Then, after performing the reduction above, this 3D theory will be coupled to the $G$ gauge group descending from the $5 \mathrm{D}$ gauge group. The resulting theory will be a $3 \mathrm{D}$ $\mathcal{N}=2$ star-shaped quiver-type theory, generalizing the $\mathcal{N}=4$ star-shaped quiver of [25]. It would be interesting to derive this also from the $S_{b}^{3} \times \Sigma_{g}$ partition function, but at present it is not known how to introduce such codimension 2 defects in this observable. Instead, in Sec. IV, we will describe how to incorporate punctures by starting with the known $\mathcal{N}=4$ star-shaped quiver of [25], which is known to be dual to the reduction of $\mathcal{N}=2$ class $\mathcal{S}$ theories, and performing certain operations on both sides of the duality to obtain more general $\mathcal{N}=2$ dualities.

\section{Global properties and higher form symmetries}

One shortcoming of the above analysis is that, since we work on $\mathbb{R}^{3}$ or $S_{b}^{3}$, we are not sensitive to issues related to the global form of the 3D gauge group, e.g., whether it is $S U(N)$ or $S U(N) / \mathbb{Z}_{N}$. This can be understood by carefully tracing the higher form symmetries of the 6D theory upon compactification. Here we focus on the $\mathcal{N}=(2,0)$ theory for concreteness, but we expect similar issues to arise for general $\mathcal{N}=(1,0)$ SCFTs.

First, we recall that for a QFT with a $q$-form symmetry, $\Gamma$, the partition function, $Z_{\omega}$, depends on a choice of a cocycle $\omega \in H^{q+1}\left(\mathcal{M}_{d} ; \Gamma\right)$, which one can think of as a choice of background $(q+1)$-form gauge field coupled to the symmetry. One may then gauge this symmetry by summing over values of this background field, introducing a "dual" background $(d-q-1)$-form gauge field, $\tilde{\omega}$, for the Pontryagin dual group, $\hat{\Gamma}$,

$$
\tilde{Z}_{\tilde{\omega}}=\frac{1}{\sqrt{|\Gamma|}} \sum_{\omega \in \Gamma} e^{i\langle\omega, \tilde{\omega}\rangle} Z_{\omega}
$$

where we define the natural pairing $\langle\omega, \tilde{\omega}\rangle=\int_{\mathcal{M}_{d}} \omega \wedge \tilde{\omega}$. Performing this operation again returns us to the original theory.

The $6 \mathrm{D} \mathcal{N}=(2,0)$ has a two-form symmetry; however, it has some subtle properties which are related to the fact that this is a relative QFT $[41,42] .{ }^{4}$ Let us take the theory of some chosen ADE type, and let $\Gamma$ be the corresponding Abelian group (i.e., the center of the corresponding simply connected Lie group). Then, as discussed in $[42,45]$, we cannot consider an arbitrary background in $H^{3}\left(\mathcal{M}_{6}, \Gamma\right)$, as above, but must first decompose

$$
H^{3}\left(\mathcal{M}_{6}, \Gamma\right) \cong L \oplus \tilde{L}
$$

where $L, \tilde{L}$ are Lagrangian subgroups, i.e., the natural pairing on $H^{3}$ vanishes on each subgroup. Now, the partition function is replaced by a "partition vector." For one choice of basis, the components can be labeled by element in $L$,

$$
Z_{\lambda}, \quad \lambda \in L \subset H^{3}\left(\mathcal{M}_{6}, \Gamma\right) .
$$

We may alternatively define

$$
\tilde{Z}_{\tilde{\lambda}}=\frac{1}{\sqrt{|L|}} \sum_{\lambda \in L} e^{i\langle\lambda, \tilde{\lambda}\rangle} Z_{\lambda}
$$

We see the two choices are essentially related by the duality mentioned above, so we might call this a "self-dual twoform symmetry." Note also that the partition function is not well defined by itself, only after making some choice of $L$ and $\lambda \in L$.

Next, suppose we compactify a $d$-dimensional theory with a $q$-form symmetry $\Gamma$ on a manifold $\mathcal{C}_{p}$. In general, we expect the compactified theory to have a $q$-form symmetry valued in $H_{0}\left(C_{p} ; \Gamma\right) \cong \Gamma$, a $(q-1)$-form symmetry valued in $H_{1}\left(C_{p} ; \Gamma\right)$, and so on up to a $(q-p)$-form symmetry valued in $H_{p}\left(C_{p} ; \Gamma\right) \cong \Gamma$. The operators which couple to these new symmetries come from the $q$-dimensional operators in the original theory partially wrapping the compactified directions; however, some of these may become very massive in the compactification limit, and the corresponding symmetries will then act trivially.

Let us now see how this works when we compactify the 6D theory to 3D. Following the philosophy of this paper, we will consider the two possible compactification orders, going through 5D or 4D, and compare the results in each case.

\footnotetext{
${ }^{4}$ See also $[43,44]$ for related issues in the context of the 3D-3D correspondence.
} 


\section{Compactifying to $5 \mathrm{D}$ first}

Here we expect to get the 5D $\mathcal{N}=2$ SYM theory with simply connected ADE group $G$. To be precise, the global form of the gauge group depends on our choice of $L$. Note that $H^{3}\left(\mathcal{M}_{5} \times S^{1}\right)$ is isomorphic to $H^{3}\left(\mathcal{M}_{5}\right) \oplus H^{2}\left(\mathcal{M}_{5}\right)$, and each term is a Lagrangian subgroup. Suppose we take $L$ to be the $H^{3}\left(\mathcal{M}_{5}\right)$ subgroup. Then the 5D partition function depends on a choice of $\omega \in H^{3}\left(\mathcal{M}_{5}\right)$, which is the same data as an ordinary two-form symmetry [43]. This is precisely the $G / \Gamma$ theory, which has such a two-form magnetic $\Gamma$ symmetry. On the other hand, if we choose $L$ to be $H^{2}\left(\mathcal{M}_{5}\right)$, we get the $G$ gauge theory, with a one-form electric $\Gamma$ symmetry. Note that if the $6 \mathrm{D}$ two-form symmetry were an ordinary one, we would have both a one- and two-form symmetry at the same time, but the self-duality implies we get only one at a time, and in fact the two choices are related by gauging. We can think of these two choices as differing by whether we wrap the threedimensional two-form charge operators of the 6D theory on the $S^{1}$, when they become the two-dimensional charge operators of the two-form magnetic symmetry in 5D, or leave them unwrapped, leading to the one-form electric symmetry charge operators.

Now, we compactify further on a Riemann surface, $\Sigma_{g}$. Then we have seen we get a star-shaped quiver, and the central node will just be the 5D gauge group. Let us first consider the $G / \Gamma$ gauge theory. Then the two-form symmetry decomposes into a 2,1 and 0-form symmetry in 3D, but we claim only the 0 -form symmetry survives in the low energy theory, becoming the magnetic $\Gamma$ symmetry of the 3D gauge theory. On the other hand, if we start with the $G$ gauge theory, we find only the one-form $\Gamma$ symmetry survives in 3D. These can also be seen directly from 6D as the cases where we wrap the charge operators on the $S^{1}$, in the first case, or on the $\Sigma_{g}$, in the second.

\section{Compactifying to $4 \mathrm{D}$ first}

Alternatively, we can first compactify on $\Sigma_{g}$, obtaining a 4D class $\mathcal{S}$ theory. Then, we can write

$$
\begin{aligned}
H^{3}\left(\mathcal{M}_{4} \times \Sigma_{g}\right)= & H^{1}\left(\mathcal{M}_{4}\right) \oplus\left(H^{1}\left(\Sigma_{g}\right) \otimes H^{2}\left(\mathcal{M}_{4}\right)\right) \\
& \oplus H^{3}\left(\mathcal{M}_{4}\right)
\end{aligned}
$$

Following [45], we may pick a Lagrangian subgroup, $K$, of $H_{1}\left(\Sigma_{g}\right)$, and this choice determines the one-form symmetry of the 4D theory, e.g., determining the global form of the gauge group. ${ }^{5}$ In addition, we should choose $L$ to include either $H^{1}\left(\mathcal{M}_{4}\right)$, leading to a zero-form $\Gamma$ symmetry, or $H^{3}\left(\mathcal{M}_{4}\right)$, leading to a two-form $\Gamma$ symmetry.

\footnotetext{
${ }^{5}$ For a genus $g$ Riemann surface, there are $g$ pairs of $(a, b)$ cycles, and one can choose $K$ by choosing one of each of these, which corresponds to taking $G$ or $G / \Gamma$ for each $\mathcal{N}=4$ loop (in an appropriate duality frame).
}

We claim the usual form of class $\mathcal{S}$ theories discussed in the literature corresponds to the former choice, and in particular, that these theories all come with a privileged $\Gamma$ zero-form symmetry. We will discuss the action of this symmetry more explicitly in the next section when we describe the 4D models in more detail. If desired, we may gauge this symmetry to obtain a new theory with a twoform symmetry, corresponding to the other choice of subgroup. These two choices correspond to taking the charge operators to be unwrapped, in the former case, or to wrap $\Sigma_{g}$, in the latter.

Now, we compactify further to 3D. If we started with the usual class $\mathcal{S}$ theory, with its zero-form symmetry, we obtain a zero-form symmetry in $3 \mathrm{D}$. We claim this is dual to the star-shaped quiver with $G / \Gamma$ gauge symmetry, as was already observed in [46]. We can see that in both cases, the 6D charge operators are wrapping only the $S^{1}$ we have reduced on. On the other hand, if we start with the twoform symmetry in $4 \mathrm{D}$, it will reduce to 2,1 and 0 form symmetries, but only the one-form symmetry survives, and the theory is dual to the star-shaped quiver with $G$ gauge symmetry.

On the other hand, the reduction of the one-form symmetry to $3 \mathrm{D}$ is more subtle. As a simple example, if we started with a genus-one Riemann surface, this may have either an electric or magnetic one-form symmetry depending on our choice of $K$ above, leading to either the $G$ $\mathcal{N}=4$ SYM theory, or the electromagnetic-dual $G / \Gamma$ theory. However, upon naive reduction to $3 \mathrm{D}$, the former theory has only a one-form symmetry, and the latter only a zero-form magnetic symmetry, even though the 4D descriptions are equivalent. This apparent contradiction, which arises more generally whenever $g>0$, is presumably related to the fact that the naive reductions correspond to "bad theories" in the terminology of [37]. It would be interesting to understand this issue in more detail.

\section{REDUCTION OF 4D CLASS $\mathcal{S}$ THEORIES}

In this section, we arrive at an alternative description of the $3 \mathrm{D}$ theories of the previous section. Namely, starting from the $6 \mathrm{D} \mathcal{N}=(2,0) \mathrm{SCFT}$, we may compactify this on a Riemann surface $\Sigma_{g}$ with flux $\mathfrak{n}$ for the $S p(1)_{F} \subset S p(2)_{R}$ symmetry to obtain a $4 \mathrm{D} \mathcal{N}=1$ theory. We may then compactify this theory on a circle to obtain a $3 \mathrm{D} \mathcal{N}=2$ theory. We expect the theory we obtain in this way to be equivalent to that obtained by first compactifying to 5D and then 3D, as in the previous section, and in this way, we may derive 3D dualities, which we consider in the following section.

\section{A. 4D models}

The theories associated to compactifications of the $6 \mathrm{D}$ $\mathcal{N}=(2,0)$ theory on a punctured Riemann surface were originally discussed in [6] in the $\mathcal{N}=2$ case, and later 
generalizations to $\mathcal{N}=1$ theories were described in $[7,8,33,34]$. We focus on the theories of type $A_{N-1}$. To briefly summarize, the ingredients specifying the compactifications are as follows:

(i) The genus, $g$, of the Riemann surface.

(ii) The number of punctures, $s$.

(iii) The Chern-degrees, $p$ and $q$, of the two line bundles describing the normal bundle of the M5 branes, constrained by

$$
p+q=2(g-1)+s .
$$

In the language of the previous section, where we took $s=0$, this corresponds to a $S p(1)_{F}$ flux $\mathfrak{n}$ of

$$
\mathfrak{n}=\frac{p-q}{2} .
$$

(iv) For each of the $s$ punctures, a choice of $S U(2)$ embedding, $\rho$, into $S U(N)$.

(v) Additionally, for each puncture, we choose a sign, separating them into + punctures and - punctures, and let $s_{ \pm}$denote the number of each type.

These theories are typically non-Lagrangian in the sense that there is no known simple Lagrangian describing the fixed point SCFT. However, in the $A_{1}$ case, we may construct explicit Lagrangians for these models from three types of building blocks, shown in Fig. 1. The basic building block is a chiral field $Q_{i j k}$ in trifundamental irreducible representation of $S U(2) \times S U(2) \times S U(2)$. This theory corresponds to a sphere with three positive punctures. We assign to this chiral field charge $\frac{1}{2}$ under the
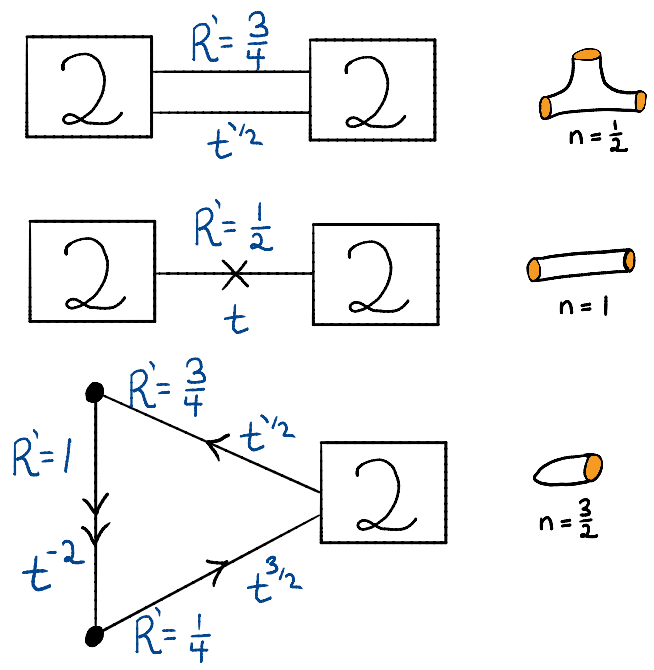

FIG. 1. The building blocks from four dimensions. The powers of $t$ denote charge under $U(1)_{t}$, and $R^{\prime}$ is the R-symmetry assignment. The double arrow for the theory on the bottom denotes having two fields of same charges. The $x$ denotes a chiral field flipping the $S U(2)^{2}$ quadratic invariant built from the bifundamental field.
Abelian global symmetry $U(1)_{t}$. We will also assign to it an $\mathrm{R}$-charge of $R^{\prime}=\frac{3}{4}$ for later convenience. This is not a superconformal R-symmetry but is useful for various computations. We also associate to the theory flux $\mathfrak{n}=\frac{1}{2}$. Two other building blocks are obtained by closing the punctures [34]. We can obtain a theory corresponding to two punctured sphere with flux one by flipping and then closing one of the punctures. We can construct a moment map operator $M_{i}{ }^{j}=Q_{i l k} Q^{j l k}$, where indices are contracted with the epsilon symbol, and we introduce a field $\Phi$ in the adjoint representation of $S U(2)$ coupled to the moment map linearly, $\operatorname{Tr} M \Phi$. Note that $\Phi$ has $R^{\prime}$ charge $+\frac{1}{2}$ and $U(1)_{t}$ charge -1 . Next, we give nilpotent expectation value to $\Phi$. This breaks the $S U(2)$ symmetry. In the IR, the theory is a Wess-Zumino (WZ) model built from a bifundamental chiral field of two $S U(2) \mathrm{s}$ and a flipper field. The bifundamental field has charge -1 under the $U(1)_{t}$ symmetry and R-charge $+\frac{1}{2}$, with the flipper fields having Rcharge $+\frac{3}{2}$ and $U(1)_{t}$ charge 2 . We can further close an additional puncture to obtain the theory corresponding to a sphere with one puncture with flux $\frac{3}{2}$. Closing punctures shifts the flux by $+\frac{1}{2}$.

The procedure of flipping a puncture changes its sign. We can close a puncture without flipping by giving expectation value to the moment map. This will shift the flux by $-\frac{1}{2}$. A general theory is obtained by gluing the blocks together. Gluing can be done [8] either by gauging diagonal $S U(2)$ symmetry of two punctures of same sign with introduction of adjoint field $\Phi$ coupled to the two moment maps, or by gluing two punctures of opposite sign turning on a superpotential coupling the two moment maps. These two procedures are consistent with the above definitions of signs of punctures.

We will discuss explicit examples of these Lagrangians below, when we consider their dimensional reduction to $3 \mathrm{D}$.

\section{B. General aspects of reduction to 3D}

We wish to reduce the four-dimensional models on a circle to three dimensions. There are several general comments we want to discuss. First, as all the symmetries in four dimensions preserved by superpotentials are not anomalous in the theories we will consider, we do not expect to generate any monopole superpotentials upon reduction [4]. Second, we want to discuss the relation between marginal and relevant operators in four and three dimensions. If we have no $U(1)$ symmetries in four dimensions and no accidental symmetries appearing upon reduction, we expect that exactly marginal operators in four dimensions become exactly marginal in three and the same for relevant operators. However, in our setup, we do have $U(1)_{t}$ symmetry. Upon reduction the superconformal R-symmetry will be in general different. Thus, the relevance of different operators might change. In particular, a 
relevant operator can become marginal and a marginal operator can become either irrelevant or relevant.

Let us discuss two examples. First, consider the model corresponding to a tube with flux one. This is a WessZumino model with cubic interactions in four dimensions and thus flows to a free theory. On the other hand, the superpotential is relevant in three dimensions and the model flows to interacting SCFT. Thus, we have an order of limits issue. If we first flow to the SCFT in four dimensions and then reduce to three, we get a free model. If we first reduce on the circle with nonvanishing superpotential and then flow, we get an interacting theory. Another example is of giving mass to the adjoint chiral fields $\Phi$. If we build general models with three punctured spheres with flux $+\frac{1}{2}$, we get theories with flux $g-1+\frac{s}{2}$, with $g$ being the genus and $s$ the number of punctures (which we take to be even). Upon giving masses to $\Phi$ we flow to a theory corresponding to same number of punctures and genus but with flux 0 . These are different models in four dimensions. The latter has quartic interactions for the fields and has a large conformal manifold not passing through zero coupling, with the former having cubic interactions and a conformal manifold passing through zero coupling. However, if we reduce the former model to three dimensions, the superconformal R-symmetry assigns charge +1 to $\Phi$ and $+\frac{1}{2}$ to $Q$ (see, e.g., [47]). This means that a quadratic superpotential for $\Phi$ is marginal, as well as the quartic $Q^{4}$ and the cubic $\Phi Q^{2}$. The first two have opposite charges under $U(1)_{t}$, and thus a combination of them is an exactly marginal deformation [48]. Thus, if we first reduce the model to three dimensions and then deform by a mass term for the adjoint we stay on the same conformal manifold. In particular, although the two theories above are different in four dimensions they sit on same conformal manifold in three dimensions. In particular, the models corresponding to different value of flux in four dimensions, 0 and $g-1+\frac{s}{2}$, correspond to same model in three dimensions.

On the other hand, because a marginal operator in four dimensions can be relevant in three dimensions, two theories residing on the same conformal manifold in four dimensions can flow to different models in three. If we have a conformal manifold in four dimensions, for example, which passes through zero coupling, the zero coupling cusp will flow to some conformal field theory (CFT) in three dimensions, but then turning on an operator which is marginal in four will be in general relevant in three dimensions and we will flow to a different model. In general, we expect that a conformal manifold in four dimensions will reduce to different CFTs. The bulk of the manifold flowing to same CFT and various cusps or loci with enhanced symmetries flowing, possibly, to different models. This is shown in Fig. 2.

These issues are important to us as we want to associate three-dimensional models to Riemann surfaces with flux.

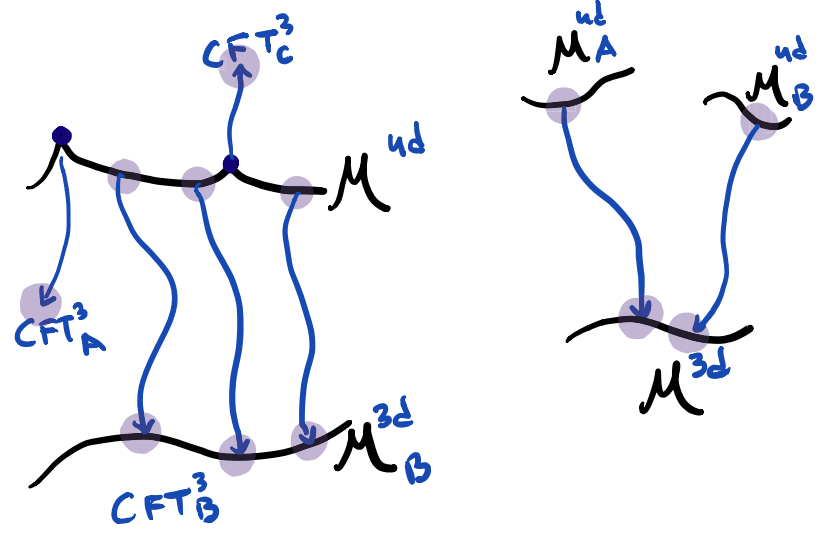

FIG. 2. Different points on same conformal manifold might flow to different theories in three dimensions. Different models in four dimensions might flow to same conformal manifold in three dimensions.

As we have understood now, we need to specify what we exactly mean by flux in three dimensions. As far as compactification from six dimension goes, the setup is that we take the $(2,0)$ theory on a Riemann surface times a circle and flow to an effective theory in three dimensions for which we then find a three-dimensional UV completion. The tunable free parameters in this setup are the relative sizes of the Riemann surface and the circle and the holonomy for the $U(1)_{t}$ symmetry. If we consider punctures, then we also have holonomies for puncture symmetries around the circle. The ambiguity we encounter is related to the choice of these parameters. For special choices, say first tuning the surface to be zero size and then the circle to zero, we might get one answer while if we keep the parameters generic and finite, flow to effective three-dimensional theory and then find UV completion, the answer can be different. In what follows, we will suggest three-dimensional models for the latter setup.

\section{DUALITY}

In the previous two sections we have discussed, the same system, the $6 \mathrm{D} A_{N-1} \mathcal{N}=(2,0)$ SCFT compactified on $\Sigma_{g} \times S^{1}$ with flux, from two different points of view, leading to two different 3D descriptions. In this section, we discuss the relation between these descriptions and provide some checks that they are indeed equivalent. We will denote the reduction first to $5 \mathrm{D}$ and then to $3 \mathrm{D}$ as duality frame $B$ and the reduction first to $4 \mathrm{D}$ and then to $3 \mathrm{D}$ as duality frame $A$. To be concrete, we will concentrate on the case of $A_{1}(2,0)$ theory reductions as in this case all the models have simple Lagrangians.

\section{A. Genus zero compactifications with no punctures}

Let us first consider the example of a sphere with no punctures and flux $\mathfrak{n}$. The two dual descriptions are shown in Fig. 3. The theory on side $B$ is given by $S U(2) / \mathbb{Z}_{2}$ gauge 
(a)

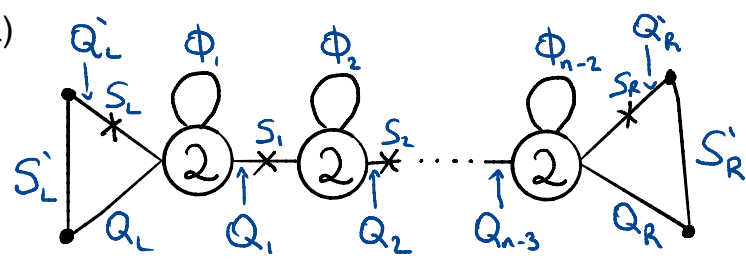

(b)

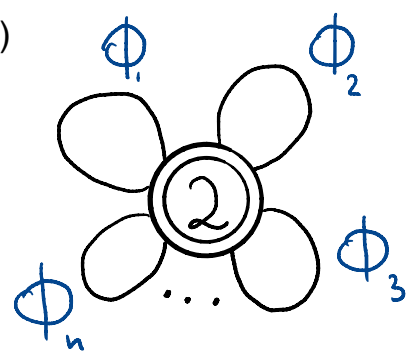

(c)

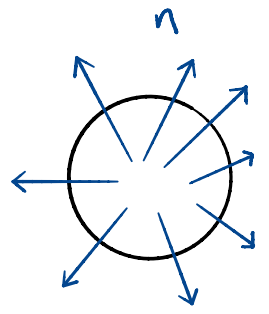

FIG. 3. Three descriptions of sphere with flux $\mathfrak{n}$. (a) is the quiver obtained by reducing on a circle the 4D theory of class $\mathcal{S}$ corresponding to compactification on a sphere with flux $\mathfrak{n}$. Here the fields $s_{i}$ are flip fields as in Fig. 1, while $s_{L}$ and $s_{R}$ are chiral fields coupling with superpotential as detailed in (35). (b) is the mirror dual, the theory obtained by reducing the 5D maximally supersymmetric Yang-Mills on a sphere with flux. Finally, (c) is the reduction geometry. Here double circle denotes gauge group $S U(2) / \mathbb{Z}_{2}$ while a circle the group $S U(2)$.

theory with $\mathfrak{n}$ adjoints. ${ }^{6}$ There is no superpotential. On side $A$ of the duality, we build the models combining together the blocks of Fig. 1. For flux $\mathfrak{n}>2$ (we will comment on $\mathfrak{n}=2$ soon), we obtain a model with $\mathfrak{n}-2 S U(2)$ gauge groups depicted in Fig. 4(a). The superpotential is

$$
\begin{aligned}
W= & \sum_{i=1}^{\mathfrak{n}-3}\left(Q_{i}^{2} \Phi_{i} \Phi_{i+1}+Q_{i}^{2} s_{i}\right)+Q_{L} \Phi_{1} Q_{L}+Q_{L}^{\prime} \Phi_{1} Q_{L}^{\prime} s_{L} \\
& +Q_{L} Q_{L}^{\prime} s_{L}^{\prime}+Q_{R} \Phi_{\mathfrak{n}-2} Q_{R}+Q_{R}^{\prime} \Phi_{\mathfrak{n}-2} Q_{R}^{\prime} s_{R} \\
& +Q_{R} Q_{R}^{\prime} s_{R}^{\prime} .
\end{aligned}
$$

Note that the fields $s_{L}, s_{L}^{\prime}, s_{R}$, and $s_{R}^{\prime}$ have the same charges. This superpotential is the most general one consistent with the charges of various fields. The charges of the various fields appear in Fig. 1 with $Q_{L, R}$ having $R^{\prime}$ charge $\frac{3}{4}, Q_{L, R}^{\prime}$ charge $\frac{1}{4}$, and $s_{L, R}, s_{L, R}^{\prime} R^{\prime}$ charge 1 . The $R^{\prime}$ charge of the adjoint fields on side $B$ is $\frac{1}{2}$, the $t$ charge is -1 , and there is no superpotential. Several comments are in order. The theory on side $B$ has a manifest $U(\mathfrak{n})$ symmetry which is not apparent on side $A$. This symmetry is conjectured to emerge in the IR. Second, the theory on

\footnotetext{
${ }^{6}$ The fact that the gauge group is $S U(2) / \mathbb{Z}_{2}$ and not $S U(2)$ is important for the duality [46]. The various partition functions one can compute depend on the global structure of the gauge groups and the difference between $S U(2) / \mathbb{Z}_{2}$ and $S U(2)$ can be easily detected leading to some discrepancies if the wrong choice is made, as was first noticed in [49].
}

(a)

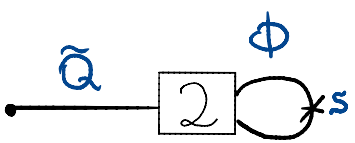

(b)

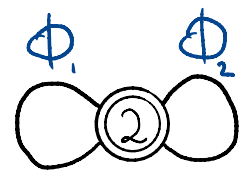

FIG. 4. Sphere with $\mathfrak{n}=2$. The theory on side (a) is a WZ model with superpotential appearing in (36).

side $B$ has a manifest $\mathbb{Z}_{2}$ symmetry under which the monopoles which have proper quantization for $S U(2) / \mathbb{Z}_{2}$ but not $S U(2)$ are charged. The symmetry is manifest also on side $A$. Note that we build theories on side $A$ from $\mathfrak{n}-1$ building blocks of Fig. 1, each containing fields charged under the gauged symmetries and gauge singlet fields. We can consider the $\mathbb{Z}_{2}$ symmetry under which the fields transforming under gauged symmetries in only one block are charged. This is exactly the $\mathbb{Z}_{2}$ symmetry we need to identify. Note that it does not matter which block is chosen as different choices are related by gauge transformations of the different $S U(2)$ gauge symmetries. Using the $\mathbb{Z}_{2}$ center of the gauge groups, we can redefine the block which is charged under the symmetry and in more generality any odd number of blocks can transform under the $\mathbb{Z}_{2}$ symmetry.

Let us discuss the map of operators. The basic operators on side $B$ of the duality are traces of quadratic combinations of the adjoints. This is in the two index symmetric representations of $U(\mathfrak{n})$. On side $A$, the operators which build this are the $\mathfrak{n}+1$ flip fields, $\mathfrak{n}-2$ adjoint quadratic, and there are $\frac{1}{2}(\mathfrak{n}-2)(\mathfrak{n}-1)$ monopole operators. The GNO charges are as follows. We have $\mathfrak{n}-2$ gauge groups with the first and last having different content than others. The charges are, $\mathfrak{n}-2$ basic monopoles $(0, \ldots, 1, \ldots)$, the monopole, $(1,1,1, \ldots, 1)$, monopoles of form $(0 \cdots, 1, \ldots, 1, \cdots)$ excluding $(1,0 \cdots, 0, \ldots, 1)$. There is no obvious symmetry acting on these operators but we claim they form the symmetric two index representation of $U(\mathfrak{n})$ symmetry conjecturally emerging in the IR.

We can perform $Z$ extremization to determine the superconformal R-symmetry. The R-symmetry increases with flux starting from slightly above $1 / 4$ for $\mathfrak{n}=2$ and approaching $1 / 2$ as flux goes to infinity. For $\mathfrak{n}>2$, there are no operators below unitarity (for $\mathfrak{n}=2$ see below). In particular, the cubic and quartic superpotentials that one can turn on are relevant deformations. All operators are above the unitarity bound. Let us now discuss a couple of examples in more detail.

\section{The case of $\mathfrak{n}=2$}

The case of $\mathfrak{n}=2$ is a bit special as it is not built by gluing together building blocks of Fig. 1 but rather by taking the sphere with one puncture and flux $\frac{3}{2}$ of that figure, flipping the puncture and closing it. The resulting theory is a WZ model in Fig. 4(a). On side $A$, we thus have the WZ model with six fields and superpotential, 


$$
W=\tilde{Q} \Phi \tilde{Q}+\mathfrak{g}^{2} \operatorname{Tr} \Phi^{2}
$$

As this is a WZ model with quartic superpotential, we need to be careful whether it is interacting and indeed $\mathfrak{g}$ goes below unitarity bound. On side $B$, it is dual to a monopole operator. On side $B$, we have supersymmetric quantum chromodynamics with gauge group $S U(2) / \mathbb{Z}_{2}=S O(3)$ and two adjoint fields. The symmetry is $U(2)$. This is the only case where we can see explicitly the symmetry on side $A$. The six fields are organized into adjoint, fundamental, and a singlet. There is a cubic superpotential between the fundamental squared and the adjoint, as well as quartic between adjoint squared and singlet squared. This is the same duality as the well-known one between $S O(N)$ and $S O\left(N_{f}-N+2\right)$ with $N=3$ and $N_{f}=2$; see [5] which is the $S O$ version of Aharony duality [50].

\section{The case of $\mathfrak{n}=3$}

Let us consider the special case of $\mathfrak{n}=3$. The theory has the same matter content as $S U(2) / \mathbb{Z}_{2} \mathcal{N}=8 \quad \mathrm{SYM}$; however, we do not turn on the cubic superpotential. The dual model is just an $S U(2)$ gauge theory with two flavors and an adjoint coupled with a quartic superpotential and four gauge singlet fields. We can consider the supersymmetric index of the two dual frames [51-53]. The index is given by

$$
\mathcal{I}=\operatorname{Tr}_{S^{2}}(-1)^{2 J_{3}} q^{\frac{1}{2}\left(E+J_{3}\right)} \prod_{i=1}^{\operatorname{rank} \mathrm{G}_{\mathrm{F}}} a_{i}^{q_{i}} .
$$

Here $J_{3}$ is the Cartan of the $S O(3)$ Lorentz symmetry, $E$ is the scaling dimension, $R$ is the R-charge, $G_{F}$ is the global symmetry group, and $q_{i}$ are the charges under the Cartan of $G_{F}$. The trace is over states in radial quantization. States contributing to the index satisfy $E-J_{3}-R=0$.

When computed in expansion in $q$ at order $q^{1}$, the index captures the marginal operators minus the conserved currents $[54,55]$. In particular, it then captures the number of exactly marginal deformations by applying the procedure of [48]. The index of the two sides of the duality at order $q$ is given by

$$
\begin{aligned}
& A: 1 \\
& B: \mathbf{1 0 - 8 - 1 .}
\end{aligned}
$$

On side $B$, we refine the index with $S U(3)$ fugacities, which we cannot do on side $A$ as this symmetry is only emergent in the IR. Here $\mathbf{8}$ stands for the character of the adjoint representation of $S U(3)$ and $\mathbf{1 0}$ for the character of the three index symmetric representation. Note that this implies that the theory has a conformal manifold which has two complex dimensions. In description $A$, this is given by the index at order $q$ plus one, as at this order the index counts marginal minus conserved current operators and the
$U(1)_{t}$ symmetry is not broken on the conformal manifold. In description $B$, we have marginal operators in $\mathbf{1 0}$ of the $S U(3)$ symmetry group. We can perform the computation of counting the dimension of the conformal manifold by counting the number of holomorphic invariants [48] built from marginal couplings which for $\mathbf{1 0}$ is two. On conformal manifold, thus the $S U(3)$ symmetry is broken and the dimension can be understood as $10-8=2$. $^{7}$

In general, we can start on side $B$ of the duality with flux $\mathfrak{n}$ and obtain any lower flux by giving masses to some of the adjoint fields. On side $A$, this cannot be done as giving mass to one of the adjoint will generate masses to all and also linear superpotentials to the flip fields and some of the monopoles. The $S U(\mathfrak{n})$ emerges in the IR and to be able to give mass only to some of the operators related by the symmetry we need to be at the fixed point.

\section{B. Adding punctures and handles}

Next, we discuss surfaces with punctures and handles. Let us first discuss side $A$ of the duality. When we add punctures, we introduce a new ingredient into the field theoretic construction. On side $A$, we consider quivers as above but we also allow some of the links between gauge groups to be trifundamental fields; see Fig. 1. Each such field will have $S U(2)$ flavor symmetry corresponding to a puncture. We have several building blocks (see Fig. 1) and we can construct same models using different blocks and combining them in different order. Each block is associated to some value of flux and as long as number of punctures and the flux are the same two theories should correspond to same IR CFT; see, for example, Fig. 4(b). Reducing these models to three dimensions, we obtain "good" theories in the nomenclature of [37]. Then to construct theories corresponding to compactifications with handles in four dimensions we just glue pairs of punctures together. Gluing corresponds as before to gauging a diagonal combination of puncture $S U(2)$ symmetries and introduction of adjoint field $\Phi$ coupled through superpotentials to moment maps. However, these theories upon reduction to three dimensions are bad. In particular, their partition functions do not converge. This is believed to be due to the fact that the superconformal R-symmetry cannot be

\footnotetext{
${ }^{7}$ Let us here mention a cute observation. We can study the theory here deformed by flipping the sextet of operators $\operatorname{Tr} \Phi_{\{i} \Phi_{j\}}$. This is a relevant deformation. Interestingly doing so we find that at order $q$ we now have $-2 \times 8-1$. This indicates that the symmetry is enhanced to $S U(3) \times S U(3) \times U(1)_{t}$. In particular, the second $S U(3)$ comes from the monopoles of $S O(3)$ which are not properly quantized for $S U(2)$. This means that the $S U(2)$ theory does not have this enhancement. The symmetry we see in the UV is the diagonal combination of the symmetries. This enhancement of symmetry is very reminiscent of the ones discussed in [56,57] in 4D where it was understood in terms of reductions of $6 \mathrm{D}$ models. It would be interesting to understand whether a similar type of explanation can be found here.
} 
identified correctly from the UV description. This is not to claim that theories corresponding to compactifications on surfaces with handles have some intrinsic problem, just that the direct reduction of the $4 \mathrm{D}$ theories is not a useful way to discuss them. A well-known example of this is the $\mathcal{N}=8$ SYM in 3D, a useful description of which is the ABJM CS/ matter theory [58]. The description $B$ we will construct will be good also in presence of handles. In particular, we will be thus able to check dualities between side $A$ and $B$ only for genus zero compactifications, albeit with any number of punctures and any value of the flux.

To consider theories on side $B$, we need to discuss a new building block, which is the well-studied $T[S U(2)]$ theory, which is a special case of the $T[S U(N)]$ theory of Gaiotto and Witten [37]. Let us start by reviewing some relevant properties of this theory, focusing in particular on the special case $N=2$. More useful observations about these models are discussed in Appendix.

\section{Properties of $T[S U(N)]$}

The $T[S U(N)]$ theory is a $3 \mathrm{D} \mathcal{N}=4$ model which describes a domain wall interpolating between $S$-dual instances of the $4 \mathrm{D} \mathcal{N}=4$ SYM theory [37]. It has $S U(N)_{H} \times S U(N)_{C}$ flavor symmetry, where we have identified the factors as the "Higgs" and "Coulomb" symmetries, in addition to the $U(1)_{t}$ symmetry of the $\mathcal{N}=2^{*}$ algebra.

There are several known descriptions of this model manifesting different subsets of the global symmetry; see Appendix. In the $N=2$ case, this model has a description as a $U(1) \mathcal{N}=4$ gauge theory with two charge one hypermultiplets. The model has manifest $\mathcal{N}=4$ supersymmetry and $S U(2)_{H} \times U(1)_{C}$ flavor symmetry. ${ }^{8}$ The $U(1)_{C}$ symmetry is the topological symmetry coming from the gauge Abelian symmetry and $S U(2)_{H}$ rotates the two hypermultiplets. It is conjectured that the $U(1)_{C}$ symmetry enhances to $S U(2)_{C}$. Thus, the symmetry in the IR is $S U(2)_{H} \times S U(2)_{C}$ and the model is self-dual under mirror symmetry. That is one $S U(2)$ rotates the Coulomb branch, and the other the Higgs branch with the two branches isomorphic. More generally, $T[S U(N)]$ has a description as a triangular quiver gauge theory, with gauge group $U(1) \times U(2) \times \ldots \times U(N-1)$, with bifundamental hypermultiplets between adjacent gauge groups, and $N$ flavors in the final $U(N-1)$ gauge groups, leading to a manifest $U(1)_{C}^{N-1} \times S U(N)_{H}$ flavor symmetry, which is enhanced in the IR to $S U(N)_{H} \times S U(N)_{C}$.

To discuss the structure of the flavor symmetry, it is convenient to schematically write the partition function on an unspecified manifold, coupling the flavor symmetries to background vector multiplets $\mathcal{V}_{H}, \mathcal{V}_{C}$, and $\mathcal{V}_{t}$, as

\footnotetext{
${ }^{8}$ The $S U(2)_{H} \times S U(2)_{C}$ flavor symmetry in the $N=2$ case should not be confused with the $\mathcal{N}=4$ R-symmetry, which we do not discuss in this subsection.
}

$$
Z_{T[S U(N)]}\left(\mathcal{V}_{H}, \mathcal{V}_{C}, \mathcal{V}_{t}\right)
$$

Then this partition function is self-dual under mirror symmetry, namely,

$$
Z_{T[S U(N)]}\left(\mathcal{V}_{H}, \mathcal{V}_{C}, \mathcal{V}_{t}\right)=Z_{T[S U(N)]}\left(\mathcal{V}_{C}, \mathcal{V}_{H},-\mathcal{V}_{t}\right)
$$

In addition, following [59], it is convenient to define a related theory called $F T[S U(N)]$, by "flipping" one of the $S U(N)$ flavor symmetries, say $S U(N)_{C}$. This means we couple an adjoint chiral multiplet to the moment map for this symmetry, which fixes this adjoint to have $U(1)_{t}$ charge -1 , and we denote this as

$$
\begin{aligned}
& Z_{F T[S U(N)]}\left(\mathcal{V}_{H}, \mathcal{V}_{C}, \mathcal{V}_{t}\right) \\
& \quad=Z_{T[S U(N)]}\left(\mathcal{V}_{H}, \mathcal{V}_{C}, \mathcal{V}_{t}\right) Z_{a d j}\left(\mathcal{V}_{C},-\mathcal{V}_{t}\right) .
\end{aligned}
$$

Then the $F T[S U(N)]$ theory is symmetric in its two flavor symmetries, without performing a $U(1)_{t}$ conjugation, i.e.,

$$
Z_{F T[S U(N)]}\left(\mathcal{V}_{H}, \mathcal{V}_{C}, \mathcal{V}_{t}\right)=Z_{F T[S U(N)]}\left(\mathcal{V}_{C}, \mathcal{V}_{H}, \mathcal{V}_{t}\right)
$$

Note this is an independent identity from (40). It can be rearranged into the following statement for the $T[S U(N)]$ theory:

$$
\begin{aligned}
& Z_{\text {adj }}\left(\mathcal{V}_{H}, \mathcal{V}_{t}\right) Z_{T[S U(N)]}\left(\mathcal{V}_{H}, \mathcal{V}_{C}, \mathcal{V}_{t}\right) Z_{\text {adj }}\left(\mathcal{V}_{C},-\mathcal{V}_{t}\right) \\
& \quad=Z_{T[S U(N)]}\left(\mathcal{V}_{H}, \mathcal{V}_{C},-\mathcal{V}_{t}\right)
\end{aligned}
$$

This says that flipping both symmetries of $T[S U(N)]$ gives the same theory up to an overall $U(1)_{t}$ conjugation [59]. Equivalently, by (40), this is the same as exchanging the two $S U(N)$ background gauge fields.

\section{2. $\mathcal{N}=2$ star-shaped quivers}

Using the $T[S U(N)]$ block, we can now state what is the dual on side $B$ of theory with flux $\mathfrak{n}$ and $s$ punctures. We claim the dual of the general 3D $\mathcal{N}=2$ class $\mathcal{S}$ theory, with data as described in Sec. III A above, is given as follows (specializing to the case of all maximal punctures, for simplicity):

(i) A central gauge group $S U(N) / \mathbb{Z}_{N}$.

(ii) This gauge group has the following ${ }^{9}$ :

$\ell$ adjoint chirals, $\Psi$, of $U(1)_{F}$ charge 1 and R-charge 1 .

$\tilde{\ell}$ adjoint chirals, $\tilde{\Psi}$, of $U(1)_{F}$ charge -1 and R-charge 1 .

$g$ adjoint chirals, $\Omega$, which have zero charge under both $U(1)_{F}$ and $U(1)_{R}$.

\footnotetext{
${ }^{9} \mathrm{We}$ recall from (14) that $U(1)_{F}$ is essentially the charge conjugation of the symmetry $U(1)$, discussed in the 4D models and their reduction above. More precisely, for $g>0$, there is an additional admixing with a hidden symmetry, but we will mostly consider the case $g=0$ below.
} 

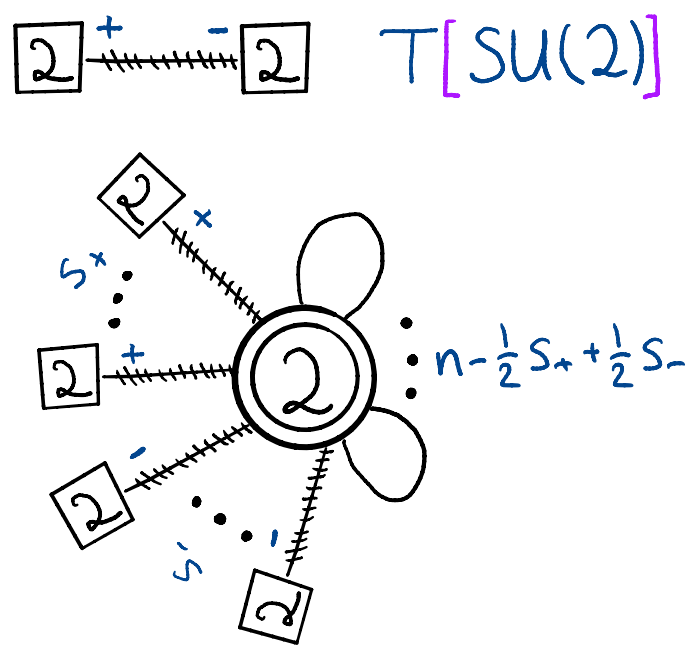

FIG. 5. Above is an abstract depiction of $T[S U(2)]$. The + and - refer, respectively, to the Coulomb and Higgs flavor symmetries of the theories. On the bottom, the sphere with flux $\mathfrak{n}$. The number of positive punctures is $s_{+}$and negative is $s_{-}$.

As in the case without punctures, we cannot in general specify $\ell, \tilde{\ell}$ individually; however, we claim their difference satisfies ${ }^{10}$

$$
\ell-\tilde{\ell}=\frac{p-q-s_{+}+s_{-}}{2} .
$$

This is because the above charges are consistent with superpotential terms of the form $\Psi \tilde{\Psi}$, which are mass terms.

(iii) For each puncture, we couple a $T[S U(N)]$ theory to this central gauge group. More precisely, for a positive puncture, we couple this to the Higgs symmetry of $T[S U(N)]$, leaving the Coulomb symmetry as a flavor symmetry, and for a negative puncture we couple to the Coulomb symmetry, leaving a Higgs flavor symmetry. For this reason, we will identify the Coulomb and Higgs symmetries of $T[S U(N)]$ as corresponding to "positive" and "negative" puncture flavor symmetries, respectively.

This is illustrated in Fig. 5 in the case $N=2$. Let us now go through several consistency checks of this formula.

(1) Zero puncture case: In the case with zero punctures and flux $\mathfrak{n}$, comparing to (17), and recalling $\mathfrak{n}=\frac{p-q}{2}$, we see this reproduces our description derived above by reduction from $5 \mathrm{D}$ as an $S U(N) / \mathbb{Z}_{N}$ gauge theory with adjoints of various $U(1)_{F}$ charges.

(2) $3 D \mathcal{N}=4$ case: In the special case,

$$
p=2(g-1)+s, \quad q=0, \quad s_{+}=s, \quad s_{-}=0,
$$

\footnotetext{
${ }^{10}$ The fact that the RHS is an integer follows from the restriction on $p$ and $q$ above. Explicitly, it can also be written as $g-1-q+s_{-}$or $1-g+p-s_{+}$.
}

the theory has $3 \mathrm{D} \mathcal{N}=4$ supersymmetry. In this case, there is a known star-shaped quiver (SSQ) "mirror" dual description [25]. This is given by an $S U(N) / \mathbb{Z}_{N}$ central node with an adjoint chiral multiplet of $U(1)_{t}$ charge 1 (in the $\mathcal{N}=4$ vector multiplet) and $g$ adjoint hypermultiplets. The latter naturally have $U(1)_{t}$ charge $-1 / 2$, but as in (13), we admix this with the $U(1)$ flavor symmetry rotating all of the hypers so that half of the adjoint chirals have charge -1 and half have charge zero. Then, we find $g$ adjoint chirals with $U(1)_{t}$ charge zero, along with $\ell=g$ of charge -1 and $\tilde{\ell}=1$ of charge 1 . Recalling that $T=-F$, we see this indeed agrees with (43) in this case, which gives

$$
\ell-\tilde{\ell}=g-1 .
$$

These are all coupled to $s$ copies of the $T[S U(N)]$ theory, which are oriented so that the "-" (Higgs) symmetries are facing inward, as above.

There is another special case which gives an $\mathcal{N}=$ 4 theory, which is

$$
p=0, \quad q=2(g-1)+s, \quad s_{+}=0, \quad s_{-}=s .
$$

Then, we see the description is much as above, except that now

$$
\ell-\tilde{\ell}=1-g,
$$

so that we may take the adjoint chirals to have opposite $U(1)_{t}$ charge. In addition, the $T[S U(N)]$ symmetries are now oriented so that their "+" (Coulomb) symmetries are facing inward, or equivalently, using (40), the Higgs symmetry faces inward but we apply a $U(1)_{t}$ conjugation. Then, using (42), this is precisely the same theory as above up to an overall $U(1)_{t}$ conjugation. This is indeed the expected result, as such a conjugation exchanges the role of $p$ and $q$ and of + and - punctures.

\section{(3) Flipping punctures}

The operation of flipping a puncture of + type (respectively, - type) corresponds to introducing a new adjoint chiral multiplet of $U(1)_{t}$ charge -1 (respectively, +1 ), which couples to the moment map of that symmetry. Suppose, we flip a + puncture in the above description. From (42), if we were to also flip the other puncture, this would be the same as reversing the orientation of the

\footnotetext{
${ }^{11}$ To avoid confusion, we work with a fixed definition of $U(1)_{t}$ on both sides of the duality, rather than mapping it with a change of sign as usual in such a mirror symmetry. Then the $U(1)_{t}$ charges in the SSQ description will have opposite sign compared to the usual conventions in the literature.
} 
$T[S U(N)]$ theory. Then this operation has the effect of reversing the $T[S U(N)]$ leg and adding a charge $T=-1$, or $F=1$, adjoint chiral to the central gauge node, which shifts

$$
\ell-\tilde{\ell} \rightarrow \ell-\tilde{\ell}+1 .
$$

But this gives precisely the expected SSQ for this new theory, which now has $\left(s_{+}, s_{-}\right) \rightarrow\left(s_{+}-1\right.$, $\left.s_{-}+1\right)$. A similar argument applies to - punctures.

(4) Gluing punctures

Next, we consider the operation of gluing punctures. For this, we first need to mention another property of the $T[S U(N)]$ theory. If we take two consecutive copies and glue them with an $\mathcal{N}=2$ vector multiplet, we find a "delta-function theory,"

$$
\begin{aligned}
& \int \mathcal{D} a_{2} Z_{T[S U(N)]}\left(a_{1}, a_{2}, \tau\right) Z_{T[S U(N)]}\left(a_{2}, a_{3}, \tau\right) \\
& \quad=\delta\left(a_{1}-a_{3}\right) .
\end{aligned}
$$

Here a delta-function theory is a formal functional of the background fields which acts as a delta function in the path integral, identifying the two gauge fields when one of them is path integrated over. This property of the $T[S U(N)]$ theory is expected on general grounds from its role as an $S$ duality wall and has been verified in certain supersymmetric partition functions $[46,49,60]$.

Now, suppose we have two SSQs, as above, and we glue a + puncture of one to a - puncture of the other. The general rules for gluing class $\mathcal{S}$ theories [7,33] tell us we should use an $\mathcal{N}=2$ vector multiplet, and then using (49), we see that the two central nodes collapse to one, which contains all of the $T[S U(N)]$ legs and adjoint chiral multiplets of the two original quivers. There are no additional adjoints introduced into the central node. Thus, we find

$$
\ell^{\text {new }}-\tilde{\ell}^{\text {new }}=\ell^{(1)}-\tilde{\ell}^{(1)}+\ell^{(2)}-\tilde{\ell}^{(2)} .
$$

This is indeed the expected result from (43), since the values of $p$ and $q$ of the two quivers simply add, and since we remove one + and one - puncture in the gluing, the difference $s_{+}-s_{-}$in (43) does not change.

If, on the other hand, we glue two + punctures, we should now gauge using a $3 \mathrm{D} \mathcal{N}=4$ vector multiplet. Then, using (40) and (42), we find

$$
\begin{aligned}
\int & \mathcal{D} a_{1} Z_{T[S U(N)]}\left(a_{1}, a_{2}, \tau\right) \\
& \times Z_{T[S U(N)]}\left(a_{1}, a_{3}, \tau\right) Z_{\text {adj }}\left(a_{1},-\tau\right) \\
& =\delta\left(a_{2}-a_{3}\right) Z_{\text {adj }}\left(a_{2},-\tau\right) .
\end{aligned}
$$

This says that if we gauge the two + punctures together with an $\mathcal{N}=4$ vector multiplet (i.e., including an additional adjoint chiral of $U(1)_{t}$ charge -1 ), then we obtain a delta-function theory with an additional adjoint chiral multiplet of $U(1)_{t}$ charge -1 . Thus, we now find

$$
\ell^{\text {new }}-\tilde{\ell}^{\text {new }}=\ell^{(1)}-\tilde{\ell}^{(1)}+\ell^{(2)}-\tilde{\ell}^{(2)}+1 .
$$

Once again, this is the expected result, since now we have reduced $s_{+}$by two. A similar statement holds for gluing two - punctures.

\section{PARTITION FUNCTION CHECKS}

In this section, we outline several computations of supersymmetric partition functions which lend further evidence to many of the dualities and relations we discussed above.

\section{A. Supersymmetric index}

Let us detail some of the supersymmetric index checks of the dualities presented above. The index is given by the trace formula (37). The technology of computing it for gauge theories has been developed in [51] and here we will follow the notations of [5]. The index of a chiral superfield is given by [46]

$\mathcal{I}_{R}(z, \mathbf{m} ; q)=\left(q^{\frac{1-R}{2}} z^{-1}\right)^{\frac{m}{2}} \prod_{i=0}^{\infty} \frac{1-(-1)^{\mathbf{m}} z^{-1} q^{\frac{1}{2}}|\mathbf{m}|+1-\frac{R}{2}+i}{1-(-1)^{\mathbf{m}} z q^{\frac{1}{2}}|\mathbf{m}|+\frac{R}{2}+i}$.

Here $R$ is the R-charge, $z$ is a fugacity for $U(1)$ symmetry under which the superfield is charged, and $\mathbf{m}$ is the magnetic flux for this symmetry through the $S^{2}$. The index of the $T[S U(2)]$ model is then given by

$$
\begin{aligned}
& \mathcal{I}(w, \mathbf{n} \mid z, \mathbf{m} ; q, t) \\
& =\mathcal{I}_{1}\left(t^{-1}, 0 ; q\right) \sum_{l \in \mathbb{Z}+\epsilon(m)} w^{2 l} \oint \frac{d h}{2 \pi i h} h^{2 \mathbf{n}} \mathcal{I}_{\frac{1}{4}}\left(t^{\frac{1}{2}}(h z)^{ \pm 1} ;\right. \\
& \quad \pm(\mathbf{l}+\mathbf{m}) ; q) \mathcal{I}_{\frac{1}{4}}\left(t^{\frac{1}{2}}\left(h^{-1} z\right)^{ \pm 1} ; \pm(\mathbf{m}-\mathbf{l}) ; q\right) .
\end{aligned}
$$

Here we use the $R^{\prime}$ R-symmetry, see Fig. 1, and $\epsilon(\mathbf{m})=\left(1-(-1)^{2 \mathbf{m}}\right) / 4$. Then the index of a sphere with $s_{+}$positive punctures, $s_{-}$negative punctures, and flux $\mathfrak{n}$ is given by 


$$
\begin{aligned}
& \mathcal{I}_{\mathfrak{n}, s_{+}, s_{-}}\left(\left\{w^{+}, w^{-}, F\right\},\left\{\mathbf{n}^{+}, \mathbf{n}^{-}\right\}, G ; q, t\right) \\
& =\sum_{\mathbf{m} \in \frac{1}{2} \mathbb{Z}} \oint \frac{d z}{4 \pi i z}\left(1-q^{|m|} z^{ \pm 2}\right) q^{-|\mathbf{m}|} G^{\frac{1-(-1)^{2} \mathbf{m}}{2}} \\
& \quad \times \prod_{i=1}^{\mathfrak{n}-\frac{1}{2} s_{+}+\frac{1}{2} s_{-}} \mathcal{I}_{\frac{1}{2}}\left(t^{-1} z^{ \pm 2} F_{i}, \pm 2 \mathbf{m} ; q\right) \mathcal{I}_{\frac{1}{2}}\left(t^{-1} F_{i}, 0 ; q\right) \\
& \quad \times \prod_{j=1}^{s_{+}} \mathcal{I}\left(w_{j}^{+}, \mathbf{n}_{j}^{+} \mid z, \mathbf{m} ; q, t^{-1}\right) \prod_{h=1}^{s_{-}} \mathcal{I}\left(w_{j}^{-}, \mathbf{n}_{j}^{-} \mid z, \mathbf{m} ; q, t\right) .
\end{aligned}
$$

Here $\left(w^{ \pm}, \mathbf{n}^{ \pm}\right)$are the fugacities and fluxes for the puncture symmetries, fugacities $F_{i}$ are for $S U(\mathbf{n})$ symmetry, and $G$ is the fugacity for the $\mathbb{Z}_{2}$ global symmetry. This index can be checked to be equal, at least in expansion in $q$, to the index computed in the dual frame using the building blocks of Fig. 1. For example, for the case of no punctures and $\mathbf{n}=3$, the index of the dual is

$$
\begin{aligned}
\sum_{m \in \mathbb{Z}} & \oint \frac{d z}{4 \pi i z}\left(1-q^{|m|} z^{ \pm 2}\right) q^{-|\mathbf{m}|} \\
& \times \mathcal{I}_{\frac{1}{2}}\left(t^{-1} z^{ \pm 2}, \pm 2 \mathbf{m} ; q\right) \mathcal{I}_{\frac{1}{2}}\left(t^{-1}, 0 ; q\right) \\
& \times \mathcal{I}_{\frac{3}{4}}\left(t^{\frac{1}{2}} z^{ \pm 1} G, \pm \mathbf{m} ; q\right) \mathcal{I}_{\frac{1}{4}}\left(t^{\frac{3}{2}} z^{ \pm 1} G, \pm \mathbf{m} ; q\right) \mathcal{I}_{1}\left(t^{-2}, 0 ; q\right)^{2} \\
& \times \mathcal{I}_{\frac{3}{4}}\left(t^{\frac{1}{2}} z^{ \pm 1}, \pm \mathbf{m} ; q\right) \mathcal{I}_{\frac{1}{4}}\left(t^{\frac{3}{2}} z^{ \pm 1}, \pm \mathbf{m} ; q\right) \mathcal{I}_{1}\left(t^{-2}, 0 ; q\right)^{2}
\end{aligned}
$$

Here the first two lines correspond to the single gauge node and the last two lines to the two one punctured spheres we glue together. The fugacity $G$ appears only in one of the two one punctured spheres. This index can be checked to be equal to $\mathcal{I}_{\mathfrak{n}=3,0,0}(\{\varnothing, \varnothing, 1\},\{\varnothing, \varnothing\}, G ; q, t)$. Note that we cannot match the $S U(\mathfrak{n})$ symmetry as this is emergent on side $A$.

\section{B. Topological index}

Next, we consider the topological index, or $\Sigma_{g} \times S^{1}$ partition function. Here we take a topological twist along $\Sigma_{g}$. We can use this index as a detailed check of some of the dualities we propose. In particular, as it is sensitive to global structure of the gauge group, it can detect some of the global issues discussed in Sec. II D.

The topologically twisted index is defined and discussed in [22-24]. Here we recall that for a gauge theory with simply connected gauge group $G$, flavor symmetry $G_{F}$, and matter in representation $R$ of $G \times G_{F}$, we introduce fugacities $x_{a}, a=1, \ldots, r_{G}$, and $\nu_{i}, i=1, \ldots, r_{G_{F}}$, associated to the Cartan subalgebras of the gauge and flavor groups, and write "Bethe equations,"12

\footnotetext{
${ }^{12}$ Here, we use the so-called " $U(1)_{-1 / 2}$ quantization" for chiral multiplets which preserves gauge invariance at the expense of introducing a parity-breaking regulator that behaves like a "level $-\frac{1}{2}$ Chern-Simons (CS) term." Below, we will implicitly shift the effective CS terms by including appropriate bare CS terms.
}

$\Pi_{a} \equiv x_{b}^{k_{a b}} \prod_{(\rho, \omega) \in R}\left(1-x^{\rho} \nu^{\omega}\right)^{-\rho_{a}}=1, \quad a=1, \ldots, r_{G}$,

where $k_{a b}$ is the matrix of (bare) Chern-Simons levels for $G$. The solutions, modulo Weyl symmetry, are in one-toone correspondence with the supersymmetric vacua of the 3D gauge theory on $\mathbb{R}^{2} \times S^{1}$. Then the $\Sigma_{g} \times S^{1}$ partition function is given by a sum over solutions to these equations of certain insertions which add handles and flavor flux on $\Sigma_{g}$.

When $G$ is not simply connected, these equations are slightly modified, as discussed in [61]. This will play an important role when mapping discrete symmetries across the duality, and we discuss this in some simple cases below.

\section{Sphere with flux $\mathfrak{n}$}

Let us consider the theory $\mathcal{T}_{3 D}\left[S_{\mathfrak{n}}{ }^{2} \times S^{1}\right]$ associated to the sphere with flux $\mathfrak{n} \geq 2$. ${ }^{13}$ Above we showed that this theory has two dual description, the star-shaped quiver description, which in this case is simply an $S O(3)$ gauge theory with $\mathfrak{n}$ fundamental chiral multiplets, and an $S U(2)^{\mathfrak{n}-2}$ linear quiver gauge theory. Here, we compare the partition functions computed using these two descriptions.

\section{Star-shaped quiver}

We first consider the star-shaped quiver description. This theory has either $S U(2)$ or $S O(3)$ gauge group, depending on the choice of higher form symmetry structure, as discussed in Sec. II D. In addition, there are $\mathfrak{n}$ adjoint chiral multiplets, with charges under $U(1)_{R}$ and $U(1)_{t}$ given in Table II. Here we note that the R-symmetry, $R^{\prime}$, of Sec. III A is related to the one obtained by reduction of the $6 \mathrm{D} U(1)_{R} \subset S p(1)_{R}$ symmetry by

$$
R^{\prime}=R+\frac{1}{2} T
$$

However, for the purposes of computing the topological index, we will require all R-charges and flavor charges to be integer quantized, and so we introduce new symmetries,

$$
\hat{R}=R^{\prime}+\frac{1}{2} T=R+T, \quad \hat{T}=2 T .
$$

We denote the fugacity for the $U(1)_{\hat{t}}$ symmetry by $\tau$.

Let us first write the Bethe equations in the case where the group is $S U(2)$,

$$
\Pi=\left(\frac{x^{2} \tau^{2}-1}{\tau^{2}-x^{2}}\right)^{2 \mathfrak{n}}=1 .
$$

Here for simplicity we have not included background

\footnotetext{
${ }^{13}$ We recall the theory with $\mathfrak{n}=0,1$ is ill-defined, and the theory with $\mathfrak{n} \rightarrow-\mathfrak{n}$ can be obtained by a $U(1)_{t}$ charge conjugation.
} 
gauge fields for the full $U(\mathfrak{n})$ flavor symmetry. This equation has $4 f$ solutions for $x$,

$$
x_{a, \pm}= \pm \sqrt{\frac{1+\tau^{2} \zeta^{a}}{\tau^{2}+\zeta^{a}}}, \quad \zeta=e^{\frac{2 \pi i}{2 n}}, \quad a=0, \ldots, 2 \mathfrak{n}-1
$$

However, when $x= \pm 1$, corresponding to $a=0$, the vacuum is lifted by fermion zero modes arising from the gauginos. Moreover, the solutions with $x \rightarrow x^{-1}$, or $a \rightarrow-a$, are related by Weyl symmetry, and so correspond to a single physical vacuum. Then, we find

$$
\mathcal{S}_{\mathrm{vac}}^{S U(2)}=\left\{x_{a, \pm}, a=1, \ldots, \mathfrak{n}-1\right\} \cup\left\{x_{\mathfrak{n},+}=i \sim x_{\mathfrak{n},-}\right\},
$$

where we note the solutions $x_{\mathfrak{n}, \pm}= \pm i$ are Weyl equivalent, and so give a single vacuum, for a total of $2 \mathfrak{n}-1$ vacua.

Next, we consider the $S O(3)$ version of the theory. The modification of the Bethe equations for nonsimply connected groups is described in [61]. In the present case, we must identify states related by large $S O(3)$ gauge transformations, which act as $x \rightarrow-x$. In addition, the Bethe equations are modified to

$$
\left(-\frac{\tau^{2} x^{2}-1}{\tau^{2}-x^{2}}\right)^{\mathfrak{n}}=\chi
$$

where $\chi \in\{ \pm 1\}$ is a fugacity for the $\mathbb{Z}_{2}^{\mathcal{J}}$ topological symmetry of the $S O(3)$ theory. Finally, in the state $x_{\mathfrak{n}}$ above, there is an unbroken $\mathbb{Z}_{2}$ gauge symmetry acting, and this $\mathbb{Z}_{2}$ gauge theory contributes two physical states, which come with $\mathbb{Z}_{2}^{\mathcal{J}}$ charges \pm 1 . To summarize, we have

$$
\mathcal{S}_{\text {vac }}^{S O(3), \chi}= \begin{cases}\left\{x_{2}, x_{4}, \ldots, x_{\mathfrak{n}-2}, x_{\mathfrak{n}}^{ \pm}\right\} & \mathfrak{n} \text { even, } \chi=1, \\ \left\{x_{1}, x_{3}, \ldots, x_{\mathfrak{n}-1}\right\} & \mathfrak{n} \text { even }, \chi=-1, \\ \left\{x_{1}, x_{3}, \ldots, x_{\mathfrak{n}-2}, x_{\mathfrak{n}}^{ \pm}\right\} & \mathfrak{n} \text { odd }, \chi=1, \\ \left\{x_{2}, x_{4}, \ldots, x_{\mathfrak{n}-1}\right\} & \mathfrak{n} \text { odd }, \chi=-1 .\end{cases}
$$

Here we dropped the subscript \pm on $x_{a, \pm}$, as we now identify these solutions, but included a superscript on $x_{\mathfrak{n}}$ accounting for the two $\mathbb{Z}_{2}$ gauge theory states, with the superscript giving their $\mathbb{Z}_{2}^{\mathcal{J}}$ charge.

Having understood the vacuum structure of the two theories, let us discuss their partition functions. These are written in terms of the handle-gluing operator and $U(1)_{\hat{t}}$ flux operator. Using the charges in Table II, we find

$$
\begin{aligned}
\mathcal{H}_{S U(2)} & =\left(x-x^{-1}\right)^{-2}\left(\left(\tau-\tau^{-1} x^{ \pm 2}\right)\left(\tau-\tau^{-1}\right)\right)^{\mathfrak{n}} H \\
\Pi_{\tau} & =\left(\left(\tau-\tau^{-1} x^{ \pm 2}\right)\left(\tau-\tau^{-1}\right)\right)^{2 \mathfrak{n}},
\end{aligned}
$$

where the Hessian, $H$, is given by
TABLE II. Charges for star-shaped quiver.

\begin{tabular}{lccccc}
\hline \hline Field & $S O(3)_{\text {gauge }}$ & $U(1)_{t}$ & $U(1)_{R}$ & $U(1)_{\hat{t}}$ & $U(1)_{\hat{R}}$ \\
\hline$\phi_{a=1}^{\mathfrak{n}}$ & 3 & -1 & 1 & -2 & 0 \\
\hline \hline
\end{tabular}

$$
H=x \frac{d \log \Pi}{d x}=\frac{4 \mathfrak{n}\left(\tau^{4}-1\right)}{\left(\tau^{2}-x^{2}\right)\left(\tau^{2}-x^{-2}\right)} .
$$

Plugging in the vacua above, we find

$$
\begin{aligned}
\mathcal{H}_{S U(2)}\left(x_{a, \pm}\right) & =\frac{4 \mathfrak{n}\left(\tau^{4}-1\right)^{3} \zeta^{a}}{\tau^{6}\left(1-\zeta^{a}\right)^{2}}\left(\frac{\left(\tau^{2}-1\right)^{3}\left(\tau^{2}+1\right)^{2} \zeta^{a}}{\tau^{3}\left(\tau^{2}+\zeta^{a}\right)\left(1+\tau^{2} \zeta^{a}\right)}\right)^{\mathfrak{n}-2} \\
\Pi_{\tau}\left(x_{a, \pm}\right) & =\left(\frac{\left(\tau^{2}-1\right)^{3}\left(1+\tau^{2}\right)^{2} \zeta^{a}}{\tau^{3}\left(\tau^{2}+\zeta^{a}\right)\left(1+\tau^{2} \zeta^{a}\right)}\right)^{2 \mathfrak{n}}
\end{aligned}
$$

For the $S O(3)$ theory, we find the same flux operator, but the handle-gluing operators are related by [61]

$\mathcal{H}_{S O(3)}=\left\{\begin{array}{cc}\frac{1}{4} \mathcal{H}_{S U(2)} & \text { trivial vacuum } \\ \mathcal{H}_{S U(2)} & \mathbb{Z}_{2} \text { gauge theory vacuum. }\end{array}\right.$

Then the partition function on $\Sigma_{g} \times S^{1}$ with $U(1)_{\hat{t}}$ flux $\mathfrak{m}$ through $\Sigma_{g}$ is given by

$$
\begin{aligned}
Z_{S U(2)}^{\Sigma_{g} \times S^{1}} & =\sum_{\hat{x} \in \mathcal{S}_{\text {vac }}^{S U(2)}} \mathcal{H}_{S U(2)}(\hat{x})^{g-1} \Pi_{\tau}(\hat{x})^{\mathfrak{m}} \\
Z_{S O(3), \chi}^{\Sigma_{g} \times S^{1}} & =\sum_{\hat{x} \in \mathcal{S}_{\text {vac }}^{S O(3), \chi}} \mathcal{H}_{S O(3)}(\hat{x})^{g-1} \Pi_{\tau}(\hat{x})^{\mathfrak{m}} .
\end{aligned}
$$

We will consider some simple examples when we compare to the dual linear $S U(2)$ quiver next. From the form of the expressions above, it suffices to find a one-to-one map between the vacua of the two theory and check that $\mathcal{H}$ and $\Pi_{\tau}$ match in dual vacua, which then implies the topological index matches for all $g$ and $\mathfrak{m}$.

\section{3. $S U(2)$ linear quiver}

As discussed in the previous section, this can be described by a linear $S U(2)$ quiver gauge theory with $\mathfrak{n}-2$ gauge nodes. Adjacent nodes are connected by a bifundamental chiral multiplet, and the two final nodes contain two fundamental chirals. Each node also contains an adjoint chiral multiplet. Finally, there are several singlet fields and superpotential couplings.

\section{4. $\mathfrak{n}=2$ case}

In this case, the theory is a Wess-Zumino model, with superpotential given by (36). In addition to the $U(1)_{t}$ and $U(1)_{R^{\prime}}$ symmetries, in the case there is an explicit $U(2)$ symmetry preserved, and the charges are shown in Table III. In addition, this theory admits a discrete $\mathbb{Z}_{2}$ 
TABLE III. Charges for $\mathfrak{n}=2$ linear quiver.

\begin{tabular}{lccccc}
\hline \hline Field & $U(2)$ & $U(1)_{t}$ & $U(1)_{R^{\prime}}$ & $U(1)_{\hat{t}}$ & $U(1)_{\hat{R}}$ \\
\hline$\tilde{Q}$ & 2 & 1 & $1 / 2$ & 2 & 1 \\
$\Phi$ & 3 & -2 & 1 & -4 & 0 \\
$\mathfrak{g}$ & 1 & 2 & 0 & 4 & 1 \\
\hline \hline
\end{tabular}

symmetry acting on $Q$ and $\mathfrak{g}$, for which we introduce a parameter $\chi \in\{ \pm 1\}$. Then there is a single vacuum, and we can immediately write down the handle-gluing and $U(1)_{\hat{t}}$ flux operators,

$$
\mathcal{H}=\left(\tau^{2}-\tau^{-2}\right)^{3} \quad \Pi_{\tau}=\frac{\left(\tau^{2}-\tau^{-2}\right)^{12}}{\left(\tau^{-1}-\chi \tau\right)^{4}\left(\tau^{-2}-\chi \tau^{2}\right)^{4}} .
$$

Let us compare this to the $\mathfrak{n}=2$ SSQ theory above. Here we identify the $\mathbb{Z}_{2}^{\mathcal{J}}$ symmetry of that theory with the $\mathbb{Z}_{2}$ symmetry discussed above. In particular, for $\chi=1$, we see from (62) that the SSQ has a single solution, with $a=2$. For $\chi=-1$, there is also a single solution, now with $a=1$. Plugging in to (65), we may compare these to (68) and find agreement. $^{14}$

\section{5. $\mathfrak{n}=3$ case}

Next, we consider the case $\mathfrak{n}=3$, which has a single $S U(2)$ gauge node with four fundamentals, an adjoint, and some singlet fields, and with superpotential given by a special case of (35). The charges are written in Table IV. Letting $x$ denote the $S U(2)$ fugacity, the Bethe equations are

$$
\begin{aligned}
\Pi & =\left(\frac{x-\tau}{1-x \tau}\right)^{2}\left(\frac{x-\tau^{3}}{1-x \tau^{3}}\right)^{2}\left(\frac{x^{2}-\tau^{-2}}{1-x^{2} \tau^{-2}}\right)^{2} \\
& =\frac{\left(x-\tau^{3}\right)^{2}(\tau x+1)^{2}}{\left(1-\tau^{3} x\right)(x+\tau)^{2}}=1,
\end{aligned}
$$

where in the second line we have canceled some factors between the numerator and denominator. Because of these cancellations, there are fewer solutions than for a theory with the same matter content but no superpotential constraints. Specifically, there is a single solution, up to Weyl invariance, at (in terms of $y=x+x^{-1}$ )

$$
y=-2\left(\tau+\tau^{-1}\right)
$$

\footnotetext{
${ }^{14}$ More precisely, we find precise matching for the eigenvalues of the handle-gluing and flux operators, but the multiplicity of these eigenvalues does not agree for $\chi=1$, i.e., we find two states on the SSQ side and only one on the WZ side. This discrepancy may be due to an additional local action for $\mathbb{Z}_{2}$ background fields, similar to that appearing in [62] in a similar context. We leave this for future investigation.
}

TABLE IV. Charges for $\mathfrak{n}=3$ quiver.

\begin{tabular}{lccccc}
\hline \hline Field & $S U(2)$ & $U(1)_{t}$ & $U(1)_{R^{\prime}}$ & $U(1)_{\hat{t}}$ & $U(1)_{\hat{R}}$ \\
\hline$q_{i=1}^{2}$ & 2 & $1 / 2$ & $3 / 4$ & 1 & 1 \\
$q_{i=3}^{4}$ & 2 & $3 / 2$ & $1 / 4$ & 3 & 1 \\
$\Phi$ & 3 & -1 & $1 / 2$ & -2 & 0 \\
$s_{a=1}^{4}$ & 1 & -2 & 1 & -4 & 0 \\
\hline \hline
\end{tabular}

To treat this cancellation carefully, we introduce a regulator, $\epsilon$, which can be thought of as a fugacity for a formal $U(1)_{\epsilon}$ symmetry which is incompatible with our choice of superpotential. This modifies the Bethe equations to

$\Pi=\frac{\left(x-e^{\epsilon} \tau\right)\left(x-e^{-\epsilon} \tau\right)}{\left(1-e^{\epsilon} x \tau\right)\left(1-e^{-\epsilon} x \tau\right)}\left(\frac{x-\tau^{3}}{1-x \tau^{3}}\right)^{2}\left(\frac{x^{2}-\tau^{-2}}{1-x^{2} \tau^{-2}}\right)^{2}=1$

To make contact with our desired theory, we must then take the limit $\epsilon \rightarrow 0$. For small but nonzero $\epsilon$, we find three vacua at

$$
\begin{aligned}
y \in\left\{-2\left(\tau+\tau^{-1}\right)+O(\epsilon),\right. \\
\\
\left.\tau+\tau^{-1} \pm \frac{i}{\sqrt{3}} \sqrt{\epsilon}\left(\tau-\tau^{-1}\right)+O(\epsilon)\right\} .
\end{aligned}
$$

We recover the solution above, plus two additional solutions, which approach $y=\tau+\tau^{-1}$ (or $x=\tau^{ \pm}$) as we remove the regulator.

Next we compute the handle-gluing and flux operators. Using the charges in Table IV, the handle-gluing operator is given by

$$
\mathcal{H}=\frac{\left(\tau-\tau^{-1}\right)\left(\tau-\tau^{-1} x^{ \pm 2}\right)\left(\tau^{2}-\tau^{-2}\right)^{4}}{\left(x-x^{-1}\right)^{2}} H
$$

where the Hessian $H$ is given by

$$
H=\frac{d \log \Pi}{d u}
$$

The behavior of $\mathcal{H}$ is smooth near the solution at $y=-2\left(\tau+\tau^{-1}\right)$, and we find

$$
\left.\mathcal{H}\right|_{y \rightarrow-2\left(\tau+\tau^{-1}\right)}=3 \frac{\left(\tau-\tau^{-1}\right)^{6}\left(\tau+\tau^{-1}\right)^{5}}{\tau^{2}+1+\tau^{-2}}
$$

For the other vacua, note that for $x \approx \tau$, we may approximate the Hessian as

$$
H \approx C+\frac{\tau+x}{\tau-x}+\frac{1}{1-e^{\epsilon} \tau x^{-1}}-\frac{1}{1-e^{\epsilon} \tau^{-1} x}
$$


This has a pole as $x$ approaches $\tau$, which competes with a zero in (71). After taking the $\epsilon \rightarrow 0$ limit carefully, we find a finite result which is the same for the two vacua,

$$
\mathcal{H}_{y \rightarrow \tau+\tau^{-1}}=3\left(\tau-\tau^{-1}\right)^{4}\left(\tau+\tau^{-1}\right)^{5} .
$$

One can similarly compute the behavior of the $U(1)_{\hat{t}}$ flux operator, and we find

$$
\Pi_{\tau}= \begin{cases}\frac{\left(\tau-\tau^{-1}\right)^{18}\left(\tau+\tau^{-1}\right)^{12}}{\left(\tau^{2}+1+\tau^{-2}\right)^{6}} & y \rightarrow-2\left(\tau+\tau^{-1}\right), \\ \left(\tau-\tau^{-1}\right)^{6}\left(\tau+\tau^{-1}\right)^{12} & y \rightarrow \tau+\tau^{-1} .\end{cases}
$$

Let us now compare to the $\mathfrak{n}=3$ case of the SSQ. Taking first $\chi=1$ there, we see there are now three vacua, one ordinary vacuum, with $a=1$, and two $\mathbb{Z}_{2}^{\mathcal{J}}$-charged vacua. Plugging in to (65), we see that the vacuum at $y=$ $-2\left(\tau+\tau^{-1}\right)$ here precisely matches with the trivial vacuum at $a=1$, while the two states approaching $y=\tau+\tau^{-1}$ match with the $\mathbb{Z}_{2}$ gauge theory contributions at $a=3$.

We can also introduce the refinement by a $\mathbb{Z}_{2}$ symmetry of this theory, which maps to the $\mathbb{Z}_{2}^{\mathcal{J}}$ topological symmetry on the SSQ side. This can be taken to act on two of the fundamental chirals, $q_{1}$ and $q_{3}$, and so, introducing a fugacity $\chi \in\{ \pm 1\}$, the Bethe equations are modified to

$$
\Pi=\frac{(x-\tau)(x-\chi \tau)\left(x-\tau^{3}\right)\left(x-\chi \tau^{3}\right)}{(1-\tau x)(1-\chi \tau x)\left(1-\tau^{3} x\right)\left(1-\chi \tau^{3} x\right)}\left(\frac{\tau^{2} x^{2}-1}{\tau^{2}-x^{2}}\right)^{2} .
$$

One may carry out the same regularization procedure as above, now taking $\chi=-1$. This time one finds a regular vacuum at $y=0$ and two degenerate vacua near $y=-\left(\tau+\tau^{-1}\right)$. However, rather than having smooth behavior as we remove the regulator, the flux and handlegluing operators now diverge in the degenerate vacuum. We take this as an indication that these vacua do not contribute, and so there is only one vacuum for $\chi=-1$. Comparing to (62), we see this agrees with the SSQ side, and taking the vacuum with $a=2$ there, we find precise agreement for the handle-gluing and flux operators.

We emphasize that the topological index is sensitive to the global form of the gauge group, and in all cases above it was crucial that we took the SSQ gauge group to be $S O(3)$, rather than $S U(2)$, both for the number of vacua to agree, and for the handle-gluing operators to precisely match, where we used the prescription of (66).

\section{Sphere with one puncture and $\mathfrak{n}=\frac{3}{2}$}

For an example involving punctures, let us consider the compactification on a sphere with flux $\mathfrak{n}=\frac{3}{2}$ and one puncture. The 4D model has a description as a WZ model, shown in Fig. 1, and the fields and charges are shown in Table V. This theory has a single vacuum. Let us consider the flavor flux operators for the $U(1)_{\hat{t}}$ and $S U(2)_{F}$ symmetries, for which we use fugacities $\tau$ and $\mu$,
TABLE V. Charges for WZ describing sphere with $\mathfrak{n}=3 / 2$ and one puncture.

\begin{tabular}{lccc}
\hline \hline Field & $S U(2)_{\mu}$ & $U(1)_{t}$ & $U(1)_{\hat{t}}$ \\
\hline$q_{1}$ & 2 & $1 / 2$ & 1 \\
$q_{2}$ & 2 & $3 / 2$ & 3 \\
$s_{a=1}^{2}$ & 1 & -1 & -2 \\
\hline \hline
\end{tabular}

respectively. In this case, the flux operators are given by

$$
\begin{aligned}
\Pi_{\mu} & =\frac{\left(\mu-\tau^{3}\right)(\mu-\tau)}{\left(1-\mu \tau^{3}\right)(1-\mu \tau)}, \\
\Pi_{\tau} & =\frac{\left(\tau^{4}-1\right)^{8}}{\left(1-\mu \tau^{3}\right)\left(1-\mu^{-1} \tau^{3}\right)(1-\mu \tau)\left(1-\mu^{-1} \tau\right)} .
\end{aligned}
$$

The dual theory is a star-shaped quiver with one $T[S U(2)]$ leg. From (43), we see we may take the central node to have a single adjoint chiral multiplet, $\Psi$, with $U(1)_{t}$ charge -1 . We find it convenient to use the dual description of the $T[S U(2)]$ theory as an $S U(2)_{k=1}$ gauge theory with two flavors, reviewed in Appendix (see Table VII for the fields and charges), as this makes the $S U(2)$ flavor symmetry manifest in the Bethe equations. Then the theory has $S U(2)_{1} \times S U(2)_{2}$ gauge symmetry, and the fields and charges are shown in Table VI. The Bethe equations are given by

$$
\begin{aligned}
& \Pi_{1}=x_{1}{ }^{2}\left(\frac{x_{1}{ }^{2} \tau^{2}-1}{\tau^{2}-x_{1}{ }^{2}}\right)^{2} \frac{\tau x_{1}-x_{2}^{ \pm} \mu^{ \pm}}{\tau-x_{1} x_{2} \mu^{ \pm}}, \\
& \Pi_{2}=x_{2}{ }^{-2} \frac{\tau x_{2}-x_{1}^{ \pm} \mu^{ \pm}}{\tau-x_{2} x_{1}{ }^{ \pm} \mu^{ \pm}} .
\end{aligned}
$$

Recall the central node of the SSQ must be taken as $S O(3)$. Equivalently, we must gauge a suitable $\mathbb{Z}_{2}$ one-form symmetry, and in the present case, one finds this acts on the solutions as

$$
\left(x_{1}, x_{2}\right) \rightarrow\left(-x_{1},-x_{2}\right) \text {. }
$$

Then, after gauging this symmetry, one finds a single solution, which can be conveniently written in terms of the Weyl and $\mathbb{Z}_{2}$ one-form-invariant quantities $y_{ \pm} \equiv y_{1} y_{2}{ }^{ \pm}$, where $y_{i}=x_{i}+x_{i}^{-1}$, as

TABLE VI. Charges for SSQ for $\mathfrak{n}=3 / 2$ and one puncture.

\begin{tabular}{lccccc}
\hline \hline Field & $S U(2)_{1}$ & $S U(2)_{2}$ & $S U(2)_{\mu}$ & $U(1)_{t}$ & $U(1)_{\hat{t}}$ \\
\hline$q$ & 2 & 2 & 2 & $-1 / 2$ & -1 \\
$\Psi$ & 3 & 1 & 1 & -1 & -2 \\
$\Theta$ & 1 & 1 & 3 & 1 & 2 \\
\hline \hline
\end{tabular}




$$
\begin{aligned}
& y_{+}=\frac{\left(1+\tau^{2}\right)(\tau-\mu)(\tau \mu-1)\left(\tau^{3}-\mu+\tau^{4} \mu+\tau^{3} \mu^{2}\right)}{\mu\left(\tau^{3}-\mu\right)\left(\tau^{3} \mu-1\right)}, \\
& y_{-}=\frac{\mu\left(\tau^{2}+1\right)}{\tau^{3}-\mu+\tau^{4} \mu+\tau^{3} \mu^{2}} .
\end{aligned}
$$

Plugging this solution into the expression for the flux operators, given by

$$
\begin{aligned}
& \Pi_{\mu}=\mu^{2}\left(\frac{\mu^{2}-\tau}{1-\tau^{2} \mu^{2}}\right)^{2} \frac{\tau \mu-x_{1}^{ \pm} x_{2}{ }^{ \pm}}{\tau-\mu x_{1}{ }^{ \pm} x_{2}{ }^{ \pm}}, \\
& \Pi_{\tau}=\frac{\left(\tau^{2}-x_{1}^{ \pm 2}\right)^{2}}{\left(1-\tau^{2} \mu^{ \pm 2}\right)^{2}}\left(\tau-x_{1}{ }^{ \pm} x_{2}{ }^{ \pm} \mu^{ \pm}\right),
\end{aligned}
$$

one finds precisely the result in (75).

\section{DISCUSSION AND COMMENTS}

In this paper, we have discussed the compactifications of the $A_{1}(2,0)$ theory in six dimensions down to three dimensions on a surface with flux times a circle. In particular, the two orders of performing such a reduction give dual theories in three dimensions. There are several ways in which this discussion can be generalized. First, we can consider the $A_{N-1}(2,0)$ for $N>2$. Here the $6 \mathrm{D} \rightarrow$ $5 \mathrm{D} \rightarrow 3 \mathrm{D}$ order of the reduction is completely analogous to what we have done. However, the $6 \mathrm{D} \rightarrow 4 \mathrm{D} \rightarrow 3 \mathrm{D}$ is more involved as the theories in 4D are, in general, currently lacking a useful description in terms of Lagrangians (however, see [63-66] for Lagrangian constructions in some cases).

Another, more interesting, venue of generalization is to compactifications of $6 \mathrm{D}$ theories with less supersymmetry. There, at least in some cases, much has been understood about the compactification from $6 \mathrm{D}$ and $4 \mathrm{D}$, and thus it is possible to use this to further compactify to $3 \mathrm{D}$. However, in these cases, the alternative $6 \mathrm{D} \rightarrow 5 \mathrm{D} \rightarrow 3 \mathrm{D}$ route has not yet been explored in detail. In particular, a very interesting question is whether a useful mirror duality can be derived by following such a route. There are several subtleties with such generalizations, however, which need to be addressed carefully. First, such a route will be most useful if we have an effective 5D gauge theory description. In general, reducing on a circle a 6D SCFT will result in a strongly coupled theory with no known such description, unless one turns on in addition some holonomy for the global symmetry around that circle. Note that for the $(2,0)$ case, no such holonomy was necessary. For example, by turning on appropriate holonomies when compactifying on a circle, 6D $(1,0)$ theories residing on M5 branes probing ADE singularities result in ADE-shaped $\mathcal{N}=1$ quiver gauge theories in $5 \mathrm{D}$. As we want to further reduce the theory on a surface of vanishing size, there can be orders of limits issues, i.e., scaling holonomies for the circle compactification with the radius of the circle and scaling the size of the surface, which should be treated carefully.

Another subtlety, in analogy to the $4 \mathrm{D}$ to $2 \mathrm{D}$ reductions discussed in [15], involves understanding the effective theory in 3D, even when the 5D Lagrangian does not involve scaling any holonomies with the radius. For example, considering minimal 6D SCFTs on a circle with a twist for discrete symmetries sometimes give a 5D gauge theory [11,67]. Compactifications of these 6D models down to $4 \mathrm{D}$ are understood and thus are a natural venue to try and extend the analysis of this paper. In the partition function language, some subtleties that can arise concern the fate of the nonzero dynamical flux sectors in the $S_{b}^{3} \times$ $\Sigma_{g}$ compactification, as well as with the role of the 5D Chern-Simons terms. A more detailed understanding of the instanton corrections may also be important for understanding these cases. We leave this for future research.

Finally, let us mention that an indirect evidence in favor of having a mirror symmetry description can be derived by studying (limits of) the superconformal index of theories obtained by compactifying the 6D theories to four dimensions. For example, taking the $(2,0)$ theory the index of theories obtained in $4 \mathrm{D}$ can be written as correlator in a topological quantum field theory (TQFT) [68] of the Riemann surface with a schematic form of [69]

$$
\mathcal{I}=\sum_{\lambda} C_{\lambda}^{2 g-2+s} \prod_{j=1}^{s} \psi_{\lambda}\left(\mathbf{a}_{j}\right) .
$$

Here $g$ is the genus of the surface and $s$ is the number of (maximal) punctures. The sum is over a certain set of parameters $\lambda$; e.g., for the type $A_{N-1}(2,0)$ theory, these parametrize finite dimensional irreducible representations of $S U(N)$. The quantity $C_{\lambda}$ is a certain function of fugacities coupled to the space-time and R-symmetries, while $\psi_{\lambda}\left(\mathbf{a}_{j}\right)$ also depend on the fugacities $\mathbf{a}_{j}$ for the global symmetry associated with $j$ th puncture. Studying the $3 \mathrm{D}$ limit of this index, which entails taking all the fugacities to 1 , the index becomes an $S^{3}$ partition function as discussed in [1-4]. Moreover, very interestingly as was shown in [60] (at least in certain limits of the fugacities), the quantities $\psi_{\lambda}(\mathbf{a})$ become the $S^{3}$ partition functions of the legs of the star-shaped quiver whereas the discrete sum with the functions $C_{\lambda}$ becomes the integration coming from the gauging of the central $S U(N) / \mathbb{Z}_{N}$ node, including the contribution of the adjoint fields. This can be viewed as an indirect indication for the existence of the star-shaped quiver mirror dual. Now, the same structure of the (limits) of the index can be derived starting from more general $6 \mathrm{D}$ theories; see, for example, [9] for the case of M5 branes probing $\mathbb{Z}_{k}$ singularities. Reducing this to three dimensions will give a star-shaped structure for the relevant $S^{3}$ partition function, which would be very interesting to understand in 
terms of a partition function of a physical theory with a starshaped structure directly in three dimensions.

\section{ACKNOWLEDGMENTS}

We would like to thank Julius Eckhard, Heeyeon Kim, Anton Nedelin, Evyatar Sabag, Sakura Schäfer-Nameki, and Gabi Zafrir for very useful conversations. We would like also to thank Sara Pasquetti for very useful comments on the draft of the paper. The research of SSR was supported by Israel Science Foundation under Grant No. 2289/18, by I-CORE Program of the Planning and Budgeting Committee, and by BSF Grant No. 2018204. B. W. was supported by the Simons Foundation Grant No. 488629 (Morrison) and the Simons Collaboration on "Special Holonomy in Geometry, Analysis and Physics."

\section{APPENDIX: DUAL DESCRIPTION OF $T[S U(N)]$}

In this Appendix, we collect some additional comments about the $T[S U(N)]$ theory. In addition to the description, we discussed in the bulk of the paper, the $T[S U(2)]$ theory has a dual description [70] which has manifest $S U(2) \times$ $S U$ (2) flavor symmetry but only $\mathcal{N}=2$ supersymmetry and no manifest time reversal symmetry. The dual is an $S U$ (2) gauge theory with Chern-Simons level one and four fundamental fields coupled through superpotential involving gauge singlet fields. The superpotential breaks the $U(4)$ symmetry to $S U(2) \times S U(2) \times U(1)$, with the two $S U(2)$ symmetries being the Coulomb and Higgs symmetries and the $U(1)$ dual to the Cartan of the enhanced R-symmetry; see Fig. 6. The fields and charges of the usual $T[S U(2)]$ description and this dual description are shown in Table VII.

One can think of the $\mathcal{N}=2$ description as follows. We can start from the three punctured sphere, which is given by a trifundamental of three $S U(2) \mathrm{s}$ associated with the punctures, and gauge one of the $S U(2)$ symmetries with level one Chern-Simons term, as shown in Fig. 7. It is well known [25] that the trifundamental theory is dual to a star-
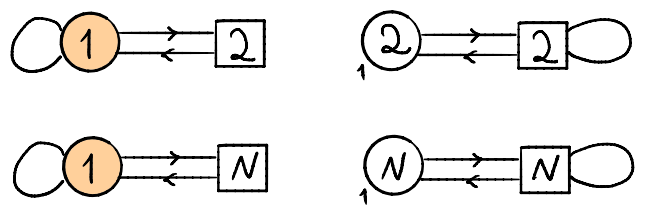

FIG. 6. The $T[S U(2)]$ theory. First, we have $\mathcal{N}=4$ description with emergent $S U(2)$ symmetry and then we have an $\mathcal{N}=2$ description with manifest $S U(2)$. The filled circles represent $U(N)$ groups, while the empty ones represent $S U(N)$. On the bottom, there is a generalization to higher rank. Here we gauge $S U(N)$ symmetry with level one of the free three punctured sphere corresponding to sphere with two maximal $S U(N)$ and a minimal $U(1)$ puncture. We have an example of enhanced supersymmetry but not symmetry there.
TABLE VII. Charges for the two dual descriptions of $T[S U(2)]$. The top table describes the usual description as $\mathcal{N}=4 U(1)$ with $N_{f}=2$, and the bottom describes the $S U(2)_{k=1}$ theory with $N_{f}=2$, which includes an additional adjoint "flip field," $\Theta$. Here we use the same $U(1)$, symmetry as in the main text, which is related by a sign to the more standard convention in the literature, and we recall $S U(2)_{H, C}$ are the Higgs and Coulomb flavor symmetries, not to be confused with the R-symmetry.

\begin{tabular}{lcccc}
\hline \hline Field & $S U(2)_{H}$ & $U(1)_{C} \subset S U(2)_{C}$ & $U(1)_{t}$ & $U(1)_{\text {gauge }}$ \\
\hline$q$ & 2 & 0 & $-1 / 2$ & 1 \\
$\tilde{q}$ & 2 & 0 & $-1 / 2$ & -1 \\
$\Phi$ & 1 & 0 & 1 & 0 \\
\hline \hline Field & $S U(2)_{H}$ & $S U(2)_{C}$ & $U(1)_{t}$ & $S U(2)_{\text {gauge }}$ \\
\hline$Q$ & 2 & 2 & $-1 / 2$ & 2 \\
$\Theta$ & 1 & 3 & 1 & 1 \\
$C S$ levels & -1 & -1 & $\cdots$ & 1 \\
\hline \hline
\end{tabular}

shaped quiver with three copies of $T[S U(2)]$ combined together by gauging a diagonal $S U(2) / \mathbb{Z}_{2}$ symmetry with $\mathcal{N}=4$ gauging. Gauging $S U(2)$ with level one ChernSimons term then in the dual theory corresponds to gauging this symmetry for one of the $T[S U(2)] \mathrm{s}$. It is easier to perform this gauging in the mirror of $T[S U(2)]$ which has manifest $S U(2)$ flavor symmetry. Using a known $U S p(2)$ duality $[50,71,72]$, this can be shown to be a topological theory equivalent to a contact term for the other $S U(2)$ with level one, tensored with a decoupled $U(1)_{2}$ topological CS theory (see also [44]), as in Fig. 8. This statement generalizes to arbitrary $T[S U(N)]$. Then one can check that the star-shaped quiver with two $T[S U(2)]$ legs with level one Chern-Simons term for the central node is actually equivalent to $T[S U(2)]$ theory with one of the puncture symmetries flipped, shown in Fig. 9. Flipping this symmetry puts the two symmetries of $T[S U(2)]$ on the same footing, meaning the model is invariant under exchanging them [73] without acting on other symmetry. Following [73], we denote the theory with one of the $S U(2)$ symmetries coupled to adjoint flip chiral fields as $F T[S U(2)]$.

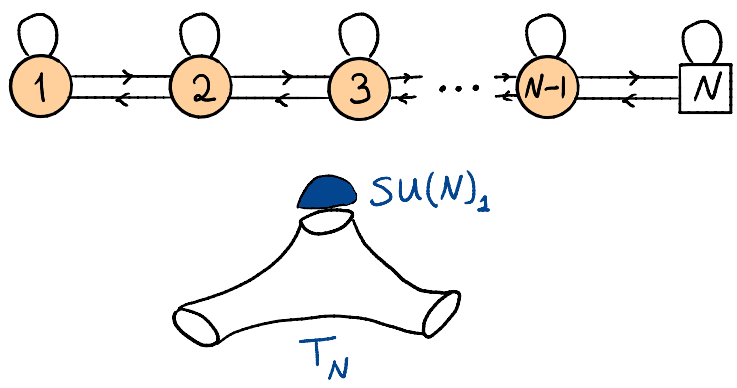

FIG. 7. The construction of the dual of $F T[S U(N)]$ theory by gauging one of the puncture symmetries. 


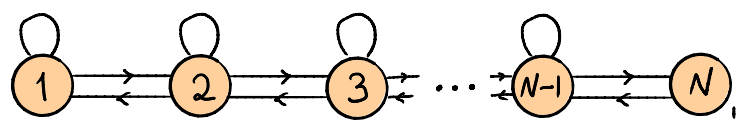

11

B.g. $\operatorname{SU}(N)_{1} \quad C S$

FIG. 8. Gauging $S U(N)$ with level one of $T[S U(N)]$ theory one obtains a TQFT which is background Chern-Simons level one theory for the other $S U(N)$. The theory also contains a decoupled TQFT sector which is not visible in index computations but can be detected by computing $S^{3}$ partition functions; see, e.g., [29] and topological indices [44].

Thus, if one believes the duality between the free trifundamental chiral field and the star-shaped quiver one derives the dual description with manifest symmetry. There is also a geometric way to understand this statement following the work of [21,74], and this can also be embedded in a more general set of dualities following [44]. The $T[S U(2)]$ theory is the three-dimensional model living on the duality wall of $\mathcal{N}=4 S U(2)$ gauge theory. From the perspective of engineering such model from the $(2,0)$ theory, this corresponds to taking the six-dimensional geometry to be four flat directions and a torus. The complex structure of the torus corresponds to the holomorphic coupling of the $\mathcal{N}=4$ gauge theory and as we have a duality wall we can think of the theory as having one of the circles nontrivially fibered in one direction of the

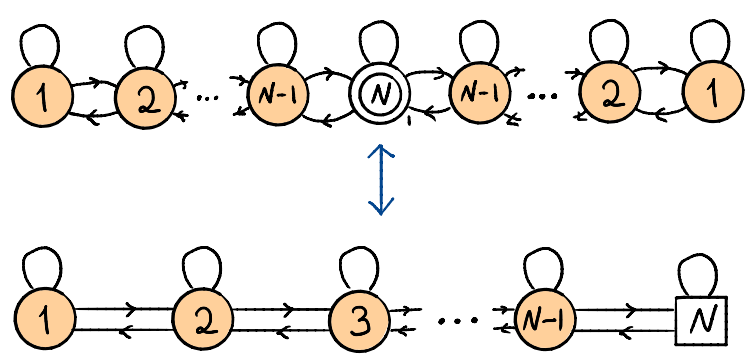

FIG. 9. The $F T[S U(N)]$ theory description using star-shaped quiver.

four-dimensional space. Such fibrations were discussed in [21] and the corresponding theories were argued to correspond to adding a Chern-Simons level to the starshaped models. In fact, introducing Chern-Simons levels when gauging symmetries of $T[S U(N)]$ was argued to correspond [37] to $T$ transformation of the duality group, then the above statement is equivalent to demanding STST $=T^{-1} S^{-1}$. This also explains why $T[S U(2)]$ is inherently three-dimensional model, as trying to make one of the circles of the four-dimensional model not compact will make it small across the domain wall.

These statements can be generalized to higher rank groups. However, at the moment, we do not have a general Lagrangian for three punctured sphere so these do not provide a better description for the $T[S U(N)]$ models.
[1] V. Niarchos, Seiberg dualities and the $3 \mathrm{~d} / 4 \mathrm{~d}$ connection, J. High Energy Phys. 07 (2012) 075.

[2] F. A. H. Dolan, V. P. Spiridonov, and G. S. Vartanov, From $4 \mathrm{~d}$ superconformal indices to $3 \mathrm{~d}$ partition functions, Phys. Lett. B 704, 234 (2011).

[3] A. Gadde and W. Yan, Reducing the $4 \mathrm{~d}$ index to the $S^{3}$ partition function, J. High Energy Phys. 12 (2012) 003.

[4] O. Aharony, S. S. Razamat, N. Seiberg, and B. Willett, 3d dualities from 4d dualities, J. High Energy Phys. 07 (2013) 149.

[5] O. Aharony, S. S. Razamat, N. Seiberg, and B. Willett, 3d dualities from $4 d$ dualities for orthogonal groups, J. High Energy Phys. 08 (2013) 099.

[6] D. Gaiotto, N = 2 dualities, J. High Energy Phys. 08 (2012) 034.

[7] F. Benini, Y. Tachikawa, and B. Wecht, Sicilian gauge theories and $N=1$ dualities, J. High Energy Phys. 01 (2010) 088.

[8] I. Bah, C. Beem, N. Bobev, and B. Wecht, Four-dimensional SCFTs from M5-Branes, J. High Energy Phys. 06 (2012) 005.

[9] D. Gaiotto and S. S. Razamat, $\mathcal{N}=1$ theories of class $\mathcal{S}_{k}$, J. High Energy Phys. 07 (2015) 073.
[10] H. C. Kim, S. S. Razamat, C. Vafa, and G. Zafrir, EString theory on Riemann surfaces, Fortschr. Phys. 66, 1700074 (2018).

[11] S.S. Razamat and G. Zafrir, Compactification of $6 \mathrm{~d}$ minimal SCFTs on Riemann surfaces, Phys. Rev. D 98, 066006 (2018).

[12] S. S. Razamat and E. Sabag, Sequences of 6d SCFTs on generic Riemann surfaces, J. High Energy Phys. 01 (2020) 086.

[13] J. J. Heckman and T. Rudelius, Top down approach to 6D SCFTs, J. Phys. A 52, 093001 (2019).

[14] O. Aharony, S. S. Razamat, and B. Willett, From 3d duality to 2d duality, J. High Energy Phys. 11 (2017) 090.

[15] A. Gadde, S. S. Razamat, and B. Willett, On the reduction of $4 \mathrm{~d} \mathcal{N}=1$ theories on $\mathbb{S}^{2}$, J. High Energy Phys. 11 (2015) 163.

[16] M. Dedushenko and S. Gukov, IR duality in $2 \mathrm{D} N=(0,2)$ gauge theory with noncompact dynamics, Phys. Rev. D 99, 066005 (2019).

[17] T. Dimofte, D. Gaiotto, and S. Gukov, Gauge theories labeled by three-manifolds, Commun. Math. Phys. 325, 367 (2014). 
[18] T. Dimofte, D. Gaiotto, and S. Gukov, 3-manifolds and 3d indices, Adv. Theor. Math. Phys. 17, 975 (2013).

[19] H. J. Chung, T. Dimofte, S. Gukov, and P. Sułkowski, 3d-3d correspondence revisited, J. High Energy Phys. 04 (2016) 140.

[20] P. M. Crichigno, D. Jain, and B. Willett, 5d partition functions with a twist, J. High Energy Phys. 11 (2018) 058.

[21] S. Gukov and D. Pei, Equivariant Verlinde formula from fivebranes and vortices, Commun. Math. Phys. 355, 1 (2017).

[22] F. Benini and A. Zaffaroni, A topologically twisted index for three-dimensional supersymmetric theories, J. High Energy Phys. 07 (2015) 127.

[23] C. Closset and H. Kim, Comments on twisted indices in $3 \mathrm{~d}$ supersymmetric gauge theories, J. High Energy Phys. 08 (2016) 059.

[24] N. A. Nekrasov and S. L. Shatashvili, Bethe/gauge correspondence on curved spaces, J. High Energy Phys. 01 (2015) 100.

[25] F. Benini, Y. Tachikawa, and D. Xie, Mirrors of 3d Sicilian theories, J. High Energy Phys. 09 (2010) 063.

[26] D. Gaiotto, G. W. Moore, and A. Neitzke, Wall-crossing, Hitchin systems, and the WKB approximation, arXiv: 0907.3987.

[27] N. Hama, K. Hosomichi, and S. Lee, SUSY gauge theories on squashed three-spheres, J. High Energy Phys. 05 (2011) 014.

[28] A. Kapustin, B. Willett, and I. Yaakov, Exact results for Wilson loops in superconformal Chern-Simons theories with matter, J. High Energy Phys. 03 (2010) 089.

[29] D. Jafferis and X. Yin, A duality appetizer, arXiv: 1103.5700 .

[30] C. Hwang and P. Yi, Twisted partition functions and $H$-saddles, J. High Energy Phys. 06 (2017) 045.

[31] C. Hwang, S. Lee, and P. Yi, Holonomy saddles and supersymmetry, Phys. Rev. D 97, 125013 (2018).

[32] L. Di Pietro and Z. Komargodski, Cardy formulae for SUSY theories in $d=4$ and $d=6$, J. High Energy Phys. 12 (2014) 031.

[33] I. Bah and B. Wecht, New $N=1$ superconformal field theories in four dimensions, J. High Energy Phys. 07 (2013) 107.

[34] P. Agarwal, K. Intriligator, and J. Song, Infinitely many $\mathcal{N}=1$ dualities from $m+1-m=1$, J. High Energy Phys. 10 (2015) 035.

[35] E. Nardoni, 4D SCFTs from negative-degree line bundles, J. High Energy Phys. 08 (2018) 199.

[36] M. Fazzi and S. Giacomelli, $\mathcal{N}=1$ superconformal theories with $D_{N}$ blocks, Phys. Rev. D 95, 085010 (2017).

[37] D. Gaiotto and E. Witten, S-duality of boundary conditions in $N=4$ super Yang-Mills theory, Adv. Theor. Math. Phys. 13, 721 (2009).

[38] A. Gadde, L. Rastelli, S. S. Razamat, and W. Yan, The 4D Superconformal Index from q-Deformed 2d Yang-Mills, Phys. Rev. Lett. 106, 241602 (2011).

[39] O. Chacaltana, J. Distler, and Y. Tachikawa, Nilpotent orbits and codimension-two defects of $6 \mathrm{~d} \mathrm{~N}=(2,0)$ theories, Int. J. Mod. Phys. A 28, 1340006 (2013).
[40] K. Yonekura, Supersymmetric gauge theory, $(2,0)$ theory and twisted 5d super-Yang-Mills, J. High Energy Phys. 01 (2014) 142.

[41] D. S. Freed and C. Teleman, Relative quantum field theory, Commun. Math. Phys. 326, 459 (2014).

[42] E. Witten, Geometric langlands from six dimensions, arXiv:0905.2720.

[43] D. Gang and K. Yonekura, Symmetry enhancement and closing of knots in $3 \mathrm{~d} / 3 \mathrm{~d}$ correspondence, J. High Energy Phys. 07 (2018) 145.

[44] J. Eckhard, H. Kim, S. Schafer-Nameki, and B. Willett, Higher-form symmetries, Bethe Vacua, and the $3 \mathrm{~d}-3 \mathrm{~d}$ correspondence, J. High Energy Phys. 01 (2020) 101.

[45] Y. Tachikawa, On the $6 \mathrm{~d}$ origin of discrete additional data of $4 \mathrm{~d}$ gauge theories, J. High Energy Phys. 05 (2014) 020.

[46] S. S. Razamat and B. Willett, Down the rabbit hole with theories of class $\mathcal{S}$, J. High Energy Phys. 10 (2014) 99.

[47] C. Bachas, I. Lavdas, and B. Le Floch, Marginal deformations of $3 \mathrm{~d} N=4$ linear quiver theories, J. High Energy Phys. 10 (2019) 253.

[48] D. Green, Z. Komargodski, N. Seiberg, Y. Tachikawa, and B. Wecht, Exactly marginal deformations and global symmetries, J. High Energy Phys. 06 (2010) 106.

[49] S. Benvenuti and S. Pasquetti, 3D-partition functions on the sphere: Exact evaluation and mirror symmetry, J. High Energy Phys. 05 (2012) 099.

[50] O. Aharony, IR duality in $\mathrm{d}=3 \mathrm{~N}=2$ supersymmetric $\mathrm{USp}(2 \mathrm{~N}(\mathrm{c}))$ and $\mathrm{U}(\mathrm{N}(\mathrm{c}))$ gauge theories, Phys. Lett. B 404, 71 (1997).

[51] S. Kim, The complete superconformal index for $\mathrm{N}=6$ Chern-Simons theory, Nucl. Phys. B821, 241 (2009); Erratum, Nucl. Phys. B864, 884 (2012).

[52] J. Kinney, J. M. Maldacena, S. Minwalla, and S. Raju, An index for 4 dimensional super conformal theories, Commun. Math. Phys. 275, 209 (2007).

[53] B. Willett, Localization on three-dimensional manifolds, J. Phys. A 50, 443006 (2017).

[54] C. Beem and A. Gadde, The $N=1$ superconformal index for class $\mathcal{S}$ fixed points, J. High Energy Phys. 04 (2014) 036.

[55] S. S. Razamat and G. Zafrir, Exceptionally simple exceptional models, J. High Energy Phys. 11 (2016) 061.

[56] S. S. Razamat, O. Sela, and G. Zafrir, Curious patterns of IR symmetry enhancement, J. High Energy Phys. 10 (2018) 163.

[57] O. Sela and G. Zafrir, Symmetry enhancement in 4d Spin(n) gauge theories and compactification from 6d, J. High Energy Phys. 12 (2019) 052.

[58] O. Aharony, O. Bergman, D. L. Jafferis, and J. Maldacena, $N=6$ superconformal Chern-Simons-matter theories, M2-branes and their gravity duals, J. High Energy Phys. 10 (2008) 091.

[59] F. Aprile, S. Pasquetti, and Y. Zenkevich, Flipping the head of $T[S U(N)]$ : Mirror symmetry, spectral duality and monopoles, J. High Energy Phys. 04 (2019) 138.

[60] T. Nishioka, Y. Tachikawa, and M. Yamazaki, 3d partition function as overlap of wavefunctions, J. High Energy Phys. 08 (2011) 003.

[61] B. Willett, Higher form symmetries in $3 \mathrm{~d} N=2$ gauge theories (to be published). 
[62] C. Cordova, P. S. Hsin, and N. Seiberg, Global symmetries, counterterms, and duality in Chern-Simons matter theories with orthogonal gauge groups, SciPost Phys. 4, 021 (2018).

[63] A. Gadde, S. S. Razamat, and B. Willett, "Lagrangian" for a Non-Lagrangian Field Theory with $\mathcal{N}=2$ Supersymmetry, Phys. Rev. Lett. 115, 171604 (2015).

[64] P. Agarwal, K. Maruyoshi, and J. Song, A Lagrangian for the $\mathrm{E}_{7}$ superconformal theory, J. High Energy Phys. 05 (2018) 193.

[65] K. Maruyoshi and J. Song, Enhancement of Supersymmetry via Renormalization Group Flow and the Superconformal Index, Phys. Rev. Lett. 118, 151602 (2017).

[66] S. S. Razamat and G. Zafrir, $N=1$ conformal dualities, J. High Energy Phys. 09 (2019) 046.

[67] P. Jefferson, H. C. Kim, C. Vafa, and G. Zafrir, Towards classification of 5d SCFTs: Single gauge node, arXiv: 1705.05836 .
[68] A. Gadde, E. Pomoni, L. Rastelli, and S. S. Razamat, S-duality and 2d Topological QFT, J. High Energy Phys. 03 (2010) 032.

[69] A. Gadde, L. Rastelli, S. S. Razamat, and W. Yan, Gauge theories and Macdonald polynomials, Commun. Math. Phys. 319, 147 (2013).

[70] J. Teschner and G. Vartanov, 6j symbols for the modular double, quantum hyperbolic geometry, and supersymmetric gauge theories, Lett. Math. Phys. 104, 527 (2014).

[71] A. Giveon and D. Kutasov, Seiberg duality in Chern-Simons theory, Nucl. Phys. B812, 1 (2009).

[72] B. Willett and I. Yaakov, $N=2$ dualities and $\mathrm{Z}$ extremization in three dimensions, arXiv:1104.0487.

[73] A. Nedelin, S. Pasquetti, and Y. Zenkevich, T[SU(N)] duality webs: Mirror symmetry, spectral duality and gauge/CFT correspondences, J. High Energy Phys. 02 (2019) 176.

[74] D. Pei, 3d-3d correspondence for Seifert manifolds, Ph.D. dissertation, California Institute of Technology, 2016. 